\title{
Effects of fast-ion-orbit width on Alfvén instabilities in stellarators: a general theory and its application to a W7-AS experiment
}

\author{
Ya. I. Kolesnichenko ${ }^{1}$, V. V. Lutsenko ${ }^{1}$, A. Weller ${ }^{2}$, A. Werner ${ }^{2}$, \\ H. Wobig ${ }^{2}$, Yu. V. Yakovenko ${ }^{1}$, J. Geiger ${ }^{2}$, and S. Zegenhagen ${ }^{2}$ \\ ${ }^{1}$ Institute for Nuclear Research, Prospekt Nauky 47, 03680 Kyiv, Ukraine \\ ${ }^{2}$ Max-Planck-Institut für Plasmaphysik, IPP-EURATOM \\ Association, D-17489 Greifswald, Germany \\ E-mail: Arthur.Weller@ipp.mpg.de
}

\begin{abstract}
A general expression for the growth rate of Alfvén instabilities driven by circulating and semi-trapped energetic ions in stellarators is derived, which generalizes that obtained in a recent work (Kolesnichenko Ya.I. et al 2004 Phys. Plasmas 11 158) by taking into account the finite orbit width of the energetic ions. It is found that the finite orbits typically reduce the growth rate, but in some cases they enhance instabilities, leading to additional resonances. The developed theory is applied to a particular shot (shot \#34723) in Wendelstein 7-AS, where Alfvénic activity had a bursting character, being strongest at the end of each burst. Although the presented analysis of this shot is not complete, it is sufficient to conclude that the finite orbit width triggers a weak instability at the initial stage of each instability burst, but it weakens a strong instability at the burst end. The latter effect makes the perturbative approach used in the paper applicable. An analysis of destabilized Alfvén eigenmodes in W7-AS precedes the stability analysis. In addition, an invariant of the particle motion in the stellarator field is derived, and the mode structure in weak-shear systems is determined.
\end{abstract}




\section{Introduction}

Finite orbit width of the energetic ions causing Alfvén instabilities can considerably influence the instability growth rate in tokamaks $[1,2,3,4]$. The effect of finite orbit width, $\Delta_{b}$, on Alfvén instabilities in stellarators is not studied yet, although one can expect that in these machines it will be even more important in some cases because of a smaller plasma radius and more complicated particle orbits. This motivated the fulfilment of the present work aimed to develop a stellarator-relevant theory for finite $\Delta_{b}$ and apply the developed theory to experiments on the Wendelstein 7-AS (W7-AS) stellarator [5]. Earlier it was shown theoretically [6, 7, 8] that because of the absence of the axial symmetry, there exist more types of Alfvén eigenmodes in stellarators than in tokamaks. This explains Alfvénic activity with frequencies well exceeding the frequency of Toroidicity-induced Alfvén eigenmodes (TAE) observed on W7-AS [9, 10] and Large Helical Device (LHD) [11, 12]. Furthermore, the lack of axial symmetry in stellarators leads to additional resonances in the wave-particle interaction, which is especially important for the destabilization of the mentioned high-frequency modes [13]. The theory developed in this work enables one to calculate the growth rates of instabilities associated with the destabilization of any eigenmodes and takes into account all the resonances possible in stellarator geometry.

A considerable part of the work deals with an analysis of a particular W7-AS shot (\#34723), where bursting Alfvénic activity accompanied by strong thermal crashes (strong drops, up to 30\%, of the plasma energy content without noticeable change of the plasma density) was observed during Neutral Beam Injection (NBI). A physical mechanism responsible for these crashes was suggested in a letter [14] and a relevant detailed theory will be published elsewhere. In this work, we restrict ourselves to an analysis aimed to identify the instability in the mentioned shot and clarify resonances of wave-particle interaction responsible for the instability. In addition, we calculate the instability growth rate with taking into account the finite orbit width of the energetic ions. Note that preliminary results of analysis of the Alfvén instability in the W7-AS shot \#34723 were reported in [15].

The structure of the work is as follows. In section 2 we derive a general expression for the instability growth rate, which generalizes the corresponding expressions of previous works $[13,16]$ (the finite magnitude of the perturbed longitudinal magnetic field was disregarded in [13] and taken into account in [16]). Section 3 contains an analysis of the W7-AS shot \#34723: we describe the experimental data (subsection 3.1), carry out equilibrium calculations, including the calculation of Alfvén continuum and Alfvén eigenmodes (subsection 3.2), and consider the destabilization of the calculated eigenmode by fast circulating ions (subsection 3.3). In section 4 we summarize the obtained results. In Appendix A the motion of the circulating particles and the particles circulating in the toroidal direction and trapped in the poloidal direction ("semitrapped" particles) is studied and an invariant of the particle guiding-centre motion in stellarators is derived. The features of the mode Fourier harmonics associated with 
the finite orbit width are considered in Appendix B. The structure of Alfvén modes in low-shear systems is considered in Appendix C.

\section{Growth rate of instabilities driven by circulating energetic ions with finite orbit width}

Treating Alfvén instabilities driven by the energetic ions perturbatively, we proceed from the following equation for the growth rate, $\gamma_{\alpha}$ (we keep only the driving part associated with the energetic ions) [13]:

$$
\gamma_{\alpha}=-\frac{2 \pi}{c^{2}} \frac{\operatorname{Re} \int \mathrm{d}^{3} x \widetilde{\boldsymbol{j}}^{\alpha} \cdot \widetilde{\boldsymbol{E}}}{\sum_{m, n} \int \mathrm{d}^{3} x \bar{v}_{A}^{-2}\left[\left|\Phi_{m, n}^{\prime}\right|^{2}+\left(m^{2} / r^{2}\right)\left|\Phi_{m, n}\right|^{2}\right]},
$$

where $\widetilde{\boldsymbol{j}}^{\alpha}$ is the energetic ion current caused by the Alfvén waves, $\boldsymbol{E}$ is the electric field, tilde labels perturbed quantities, $\Phi_{m, n}$ is a harmonic of the scalar potential of the perturbed electric field $(\widetilde{\Phi}), \Phi_{m, n}$ is defined by

$$
\widetilde{\Phi}(r, \vartheta, \varphi)=\sum_{m, n} \Phi_{m, n}(r) \exp (i m \vartheta-i n \varphi-i \omega t)
$$

$\omega$ is the mode frequency, $\vartheta$ and $\varphi$ are the Boozer poloidal and toroidal angles, respectively, $r$ is the radial coordinate defined by $\psi=\bar{B} r^{2} / 2$ with $\psi$ the toroidal magnetic flux, $\bar{B}$ is the average magnetic field at the magnetic axis, $\Phi^{\prime}=\mathrm{d} \Phi / \mathrm{d} r, \bar{v}_{A}$ is the Alfvén velocity taken with the magnetic field $\bar{B}$.

The current $\widetilde{\boldsymbol{j}}^{\alpha}$ can be expressed in terms of the disturbed distribution function of the energetic ions, $\widetilde{F}$, as $\widetilde{\boldsymbol{j}}^{\alpha}=e \int \mathrm{d}^{3} v \boldsymbol{v} \widetilde{F}$. The product $\widetilde{\boldsymbol{j}}^{\alpha} \cdot \widetilde{\boldsymbol{E}}$ is then (the effects of finite Larmor radius are neglected in $\widetilde{F}$ )

$$
\widetilde{\boldsymbol{j}}^{\alpha} \cdot \widetilde{\boldsymbol{E}}=c M \int \mathrm{d}^{3} v_{g c}\left(v_{\|}^{2}+0.5 v_{\perp}^{2}\right) \mathcal{K} \cdot \widetilde{\boldsymbol{E}} \widetilde{F}
$$

where $\mathrm{d}^{3} v_{g c}=(2 \pi / \mathrm{d} \alpha) \mathrm{d}^{3} v$ is the guiding centre volume element in the velocity space, $\alpha$ is the phase of the particle Larmor rotation, $\mathcal{K}=(\boldsymbol{b} \times \boldsymbol{\kappa}) / B, \boldsymbol{\kappa}$ is the field line curvature, $\boldsymbol{b}=\boldsymbol{B} / B$. When writing equation (3), we have taken into account the fact that $[16]$

$$
\int \mathrm{d} \alpha \boldsymbol{v} \cdot \widetilde{\boldsymbol{E}}=2 \pi \omega_{B}^{-1}\left(v_{\|}^{2}+0.5 v_{\perp}^{2}\right)(\boldsymbol{b} \times \boldsymbol{\kappa}) \cdot \widetilde{\boldsymbol{E}}
$$

where $\omega_{B}=e B /(M c)$.

The perturbed distribution function in equation (3) is determined by a corresponding kinetic equation. The mentioned equation has the following solution:

$$
\widetilde{F}=-\frac{e}{M} \int_{-\infty}^{t} \mathrm{~d} \tau \oint \frac{\mathrm{d} \alpha}{2 \pi}\left(\widetilde{\boldsymbol{E}}+\frac{1}{c} \boldsymbol{v} \times \widetilde{\boldsymbol{B}}\right) \cdot \frac{\partial F_{0}}{\partial \boldsymbol{v}},
$$

where the integral over $\tau$ is taken along the particle orbits, $F_{0} \equiv F_{0}\left[C_{1}(\boldsymbol{v}), C_{2}(\boldsymbol{v}), C_{3}(\boldsymbol{v})\right]$ is the unperturbed distribution function, $C_{j}(\boldsymbol{v})$ with $\mathrm{j}=1,2,3$, are the constants of the particle motion. 
Thus, a question arises what are the constants of motion in stellarators. In contrast to tokamaks, the equilibrium magnetic field in stellarators contains many Fourier harmonics, and furthermore, non-axisymmetric harmonics typically dominate. Nevertheless, because the number of the field periods along the large azimuth of the torus, $N$, well exceeds unity (e.g., $N=5$ in Wendelstein 7-AS and Wendelstein 7-X, $N=10$ in the Large Helical Device), the particle bounce/transit period associated with non-axisymmetric harmonics is rather small, which implies that the maximum radial excursion of the particle is determined mainly by the toroidicity (see Appendix A). Therefore, like for tokamaks, we take $F_{0}=F_{0}\left(P_{\varphi}, \mathcal{E}, \lambda\right)$, where $P_{\varphi}=\Psi_{p}-v_{\varphi} B / \omega_{B}$ is the toroidal angular momentum, $\Psi_{p}$ is the poloidal magnetic flux, $v_{\varphi}$ is a covariant component of the particle velocity, $\mathcal{E}$ the particle energy, $\lambda=\mu_{p} \bar{B} / \mathcal{E}, \mu_{p}$ the magnetic moment. Then, using equations (5) and (4), we obtain $\widetilde{F}$ in the form:

$$
\widetilde{F}=-c \hat{\Pi} F_{0} \int_{-\infty}^{t} \mathrm{~d} \tau\left(v_{\|}^{2}+0.5 v_{\perp}^{2}\right) \mathcal{K} \cdot \widetilde{\boldsymbol{E}},
$$

where

$$
\hat{\Pi}=M \frac{\partial}{\partial \mathcal{E}}-\frac{M \lambda}{\mathcal{E}} \frac{\partial}{\partial \lambda}+\frac{n}{\bar{r} \omega \omega_{B} \iota} \frac{\partial}{\partial \bar{r}},
$$

$\bar{r}$ is the radius of the circular orbits (in particular, $\bar{r}$ represents the orbit-averaged radial coordinate $r$ of the circulating particles with standard orbits). Equation (6) is written in the assumption that the particle orbit width is small compared to $\bar{r}$.

Putting equation (6) into equation (3), we obtain:

$$
\begin{aligned}
\widetilde{\boldsymbol{j}}^{\alpha} \cdot \widetilde{\boldsymbol{E}} & =-M c^{2} \int \mathrm{d}^{3} v\left(v_{\|}^{2}+0.5 v_{\perp}^{2}\right) \hat{\Pi} F_{0} \mathcal{K} \cdot \widetilde{\boldsymbol{E}} \\
& \times \int_{-\infty}^{t} \mathrm{~d} \tau\left(v_{\|}^{2}+0.5 v_{\perp}^{2}\right) \mathcal{K}(\tau) \cdot \widetilde{\boldsymbol{E}}(\tau) .
\end{aligned}
$$

Let us assume first that the particles encircle the magnetic axis. Then we can write the following equations of the particle motion (see Appendix A):

$$
\begin{aligned}
& r=\bar{r}+\Delta_{b} \cos \vartheta \\
& \vartheta(\tau)=\vartheta+\omega_{b}(\tau-t)-\left.\frac{\epsilon_{t}^{\prime}}{\epsilon_{t}} \Delta_{b}[\sin \vartheta(\tau)-\sin \vartheta]\right|_{\vartheta(\tau)=\vartheta+\omega_{b}(\tau-t)}, \\
& \varphi(\tau)=\varphi+\omega_{\varphi}(\tau-t),
\end{aligned}
$$

where $\omega_{b}=v_{\|} \iota / R_{0}$ is the bounce frequency, $R_{0}$ is the magnetic axis length divided by $2 \pi$, $\iota$ is the rotational transform, $\omega_{\varphi}=v_{\|} / R_{0}$ is the frequency of the particle motion in the toroidal direction, $\Delta_{b}=r_{B \|} \epsilon_{t} R_{0} /(\iota r)$ is the orbit half-width, $r_{B \|}=v_{\|} / \omega_{B}, \epsilon_{t}^{\prime}=\mathrm{d} \epsilon_{t} / \mathrm{d} r$. Due to equation (9), we can expand $\Phi_{m, n}(r)$ into a Fourier series with respect to $\vartheta$ :

$$
\Phi_{m, n}[r(\vartheta)]=\sum_{l} \Phi_{m, n, l}(\bar{r}) e^{i l \vartheta}
$$


where

$$
\Phi_{m, n, l}(\bar{r})=\frac{1}{\pi} \int_{r_{\min }}^{r_{\max }} \mathrm{d} r \Phi_{m, n}(r) \frac{\cos \left(l \cos ^{-1} \frac{r-\bar{r}}{\Delta_{b}}\right)}{\sqrt{\Delta_{b}^{2}-(r-\bar{r})^{2}}},
$$

$r_{\min }$ and $r_{\max }$ are, respectively, the minimum and maximum radial coordinates on the particle orbit, $r_{\min }=\bar{r}-\left|\Delta_{b}\right|, r_{\max }=\bar{r}+\left|\Delta_{b}\right|$ (see also Appendix B). In addition, we expand the curvature into a Fourier series, neglecting the variation of $\boldsymbol{\kappa}(r)$ on the orbit width:

$$
\kappa_{\alpha}(r)=\frac{1}{2} \sum_{\mu, \nu=-\infty}^{\infty} \kappa_{\alpha ; \mu, \nu}(\bar{r}) e^{i \mu \vartheta-i \nu N \varphi} .
$$

where $\kappa_{\alpha}$ with $\alpha=\psi, \vartheta, \varphi$ are the corresponding covariant components of the curvature vector. Note that because the curvature is a real quantity, $\kappa_{\alpha ;-\mu,-\nu}=\kappa_{\alpha ; \mu, \nu}^{*}$, where the asterisk means complex conjugation. Similarly, $\Phi_{-m,-n,-l}=\Phi_{m, n, l}^{*}$ when we take $\omega_{-m,-n}=-\omega_{m, n}$.

For Alfvén waves we can take $\widetilde{E}_{\|}=0$ and $\widetilde{\boldsymbol{A}}_{\perp}=0$, where $\boldsymbol{A}$ is the vector potential, which leads to $\widetilde{E}=-\nabla_{\perp} \widetilde{\Phi}$. On the other hand, the third covariant component of the magnetic field dominates. Taking this into account, we obtain:

$$
\mathcal{K} \cdot \widetilde{\boldsymbol{E}} \approx \kappa_{\vartheta} \frac{\partial \widetilde{\Phi}}{\partial \psi}-\kappa_{\psi} \frac{\partial \widetilde{\Phi}}{\partial \vartheta} .
$$

Equations (2), (10)-(12), and (15) enable us to write the product $\mathcal{K}(\tau) \cdot \widetilde{\boldsymbol{E}}(\tau)$ in the form:

$$
\begin{aligned}
& \mathcal{K}(\tau) \cdot \widetilde{\boldsymbol{E}}(\tau)=\frac{1}{2 \bar{B} r} \sum_{m, n, l, \mu, \nu, q, q^{\prime}} J_{q}\left(\xi_{m l \mu}\right) J_{q^{\prime}}\left(\xi_{m l \mu}\right) \\
& \times\left[\kappa_{\vartheta ; \mu, \nu} \Phi_{m, n, l}^{\prime}+i(m+l) \kappa_{r ; \mu, \nu} \Phi_{m, n, l}\right] \\
& \times \exp \left[i\left(m+l+\mu+q^{\prime}-q\right) \vartheta-i(n+\nu N) \varphi-i \omega t+i \Omega_{m l \mu q}^{n \nu}(\tau-t)\right],
\end{aligned}
$$

where $\Phi_{m, n, l}^{\prime}=\mathrm{d} \Phi / \mathrm{d} r, \kappa_{r}=\bar{B} r \kappa_{\psi}, \kappa_{\vartheta}=i \mu \epsilon_{B}^{(\mu \nu)} / 2, \epsilon_{B}^{(\mu \nu)}$ is defined in Appendix, $J_{q}\left(\xi_{m l \mu}\right)$ is the Bessel function, $\xi_{m l \mu}=(m+l+\mu) \Delta_{b} \epsilon_{t}^{\prime} / \epsilon_{t}, \Omega_{m l \mu q}^{n \nu}=(m+l+\mu-q) \omega_{b}-(n+\nu N) \omega_{\varphi}-\omega$.

When deriving equation (16), we used the expansion

$$
e^{i \xi \sin \vartheta}=\sum_{q} J_{q}(\xi) e^{i q \vartheta}
$$

Assuming that the particles are well circulating, we take $v_{\|}(\tau)=$ const and $v_{\|}^{2}(\tau)+$ $0.5 v_{\perp}^{2}(\tau)=$ const. Then, using equation (16), we can easily calculate the integral over $\tau$ in equation (8) for $\widetilde{\boldsymbol{j}}^{\alpha} \cdot \widetilde{\boldsymbol{E}}$. Another quantity in equation $(8), \boldsymbol{\mathcal { K }} \cdot \widetilde{\boldsymbol{E}}$, can be written as

$$
\begin{aligned}
& \mathcal{K} \cdot \widetilde{\boldsymbol{E}}=\frac{1}{2 \bar{B} \bar{r}} \sum_{m^{\prime}, n^{\prime}, l^{\prime}, \mu^{\prime}, \nu^{\prime}}\left[\kappa_{\vartheta ; \mu^{\prime}, \nu^{\prime}}^{*} \Phi_{m^{\prime}, n^{\prime}, l^{\prime}}^{* \prime}-i\left(m^{\prime}+l^{\prime}\right) \kappa_{r ; \mu^{\prime}, \nu^{\prime}}^{*} \Phi_{m^{\prime}, n^{\prime}, l^{\prime}}^{*}\right] \\
& \times \exp \left[-i\left(m^{\prime}+l^{\prime}+\mu^{\prime}\right) \vartheta+i\left(n^{\prime}+\nu^{\prime} N\right) \varphi+i \omega t\right] .
\end{aligned}
$$


Due to equations (16) and (18), we can calculate $\int \mathrm{d}^{3} x \widetilde{\boldsymbol{j}}^{\alpha} \cdot \widetilde{\boldsymbol{E}}$. After integration over $\vartheta$ and $\varphi$ in $\mathrm{d}^{3} x=\mathrm{d} \bar{r} \bar{r} \mathrm{~d} \vartheta \mathrm{d} \varphi$ we have:

$$
\begin{aligned}
\int \mathrm{d}^{3} x \widetilde{\boldsymbol{j}}^{\alpha} \cdot \widetilde{\boldsymbol{E}} & =-\frac{\pi^{2} M c^{2}}{\bar{B}^{2}} \int \mathrm{d} \bar{r} \bar{r}^{-1} \int \mathrm{d}^{3} v\left(v_{\|}^{2}+0.5 v_{\perp}^{2}\right)^{2} \\
& \times \sum_{m, n, l, \mu, \nu, q, m^{\prime}, n^{\prime}, l^{\prime}, \mu^{\prime}, \nu^{\prime}, q^{\prime}} J_{q}\left(\xi_{m l \mu}\right) J_{q^{\prime}}\left(\xi_{m l \mu}\right) \\
& \times \frac{\hat{\Pi} F_{0}}{i \Omega_{m l \mu q}^{n \nu}}\left[\kappa_{\vartheta ; \mu, \nu} \Phi_{m, n, l}^{\prime}+i(m+l) \kappa_{r ; \mu, \nu} \Phi_{m, n, l}\right] \\
& \times\left[\kappa_{\vartheta ; \mu^{\prime}, \nu^{\prime}}^{*} \Phi_{m^{\prime}, n^{\prime}, l^{\prime}}^{* \prime}-i\left(m^{\prime}+l^{\prime}\right) \kappa_{r ; \mu^{\prime}, \nu^{\prime}}^{*} \Phi_{m^{\prime}, n^{\prime}, l^{\prime}}^{*}\right]
\end{aligned}
$$

where

$$
m+l+\mu-q=m^{\prime}+l^{\prime}+\mu^{\prime}-q^{\prime}, \quad n+\nu N=n^{\prime}+\nu^{\prime} N .
$$

Taking $\operatorname{Im}\left(\Omega^{-1}\right)=-\pi \delta(\Omega)$ and using equations (1), (19), (20), we obtain the growth rate as follows:

$\gamma=\frac{\pi^{2} M \sum \int \mathrm{d} \bar{r} \bar{r}^{-1} \int \mathrm{d}^{3} v_{g c}\left(v_{\|}^{2}+0.5 v_{\perp}^{2}\right)^{2} \hat{\Pi} F_{0} \delta\left(\Omega_{m l \mu q}^{n \nu j}\right) J_{q}\left(\xi_{m l \mu}\right) J_{q^{\prime}}\left(\xi_{m l \mu}\right) G_{m l \mu}^{n \nu}\left[G_{m^{\prime} l^{\prime} \mu^{\prime}}^{n^{\prime} \nu^{\prime}}{ }_{(21,)}^{*}\right)}{2 \bar{B}^{2} \sum_{m, n} \int \mathrm{d} r r \bar{v}_{A}^{-2}\left[\left|\Phi_{m, n}^{\prime}\right|^{2}+\left(m^{2} / r^{2}\right)\left|\Phi_{m, n}\right|^{2}\right]}$

where $\sum \equiv \sum_{m, n, l, \mu, \nu, q, n^{\prime}, l^{\prime}, \mu^{\prime}, \nu^{\prime}, q^{\prime}, j}, j= \pm 1, \xi_{m l \mu}=(m+l+j \mu) \Delta_{b} \epsilon_{t}^{\prime} / \epsilon_{t} \approx(m+l+j \mu) \Delta_{b} / r$,

$$
\begin{aligned}
& \Omega_{m l \mu q}^{n \nu}=(m+l+j \mu-q) \omega_{b}-(n+j \nu N) \omega_{\varphi}-\omega, \\
& G_{m l \mu}^{n \nu j}=\left[(m+l) \kappa_{r ; \mu, \nu} \Phi_{m, n, l}+j \mu \epsilon_{B}^{(\mu \nu)} \Phi_{m, n, l}^{\prime}\right]
\end{aligned}
$$

$m^{\prime}=m+l-l^{\prime}+j\left(\mu-\mu^{\prime}\right)-q+q^{\prime}, n^{\prime}=n+j\left(\nu-\nu^{\prime}\right) N$, the sign of $\mu$ is fixed. Note that we changed the notation of $\xi_{m l \mu}$ and $\Omega_{m l \mu q}^{n \nu}$ by adding $j= \pm 1$. The reason is that equation (A.4) employs the magnetic field in the form

$$
B=\bar{B}\left[1+\epsilon_{0}(r)+\sum_{\mu, \nu \neq 0} \epsilon_{B}^{(\mu \nu)} \cos (\mu \vartheta-\nu N \varphi)\right]
$$

where $\epsilon_{0}(r)=\epsilon^{(00)} / 2$ and the sign of $\mu$ is fixed [before this we used the magnetic field given by equation (A.4)]. Equation (24) will be also used below.

It follows from equations (21) and (22) that the resonance condition for a mode with the mode numbers $m_{0}, n_{0}$ is

$$
\omega=\left[\left(m_{0}+l+j \mu-q\right) \iota-n_{0}-j \nu N\right] \frac{v_{\|}^{\text {res }}}{R_{0}},
$$

where $v_{\|}^{\text {res }}$ is the resonant velocity. Equation (25) shows that the finite orbit width increases the number of the resonant particles (due to $l \neq 0$ and $q \neq 0$ ). However, it typically involves low-energy particles into the interaction with the wave, which 
decreases the growth rate. On the other hand, the resonances with $l \neq 0$ and $q \neq 0$ can provide the wave-particle interaction leading to instabilities when the resonant particles in the zero-orbit-width approximation are absent (in particular, this was the case in the beginning of the instability burst in W7-AS shot \#34723, see section 3 and the region $\omega>43 \mathrm{kHz}$ in figure 11).

It is of importance to know the dependence of $\gamma$ on the mode numbers. This dependence can be found by means of a simple analysis for the well-localized modes, $\Delta_{m} \ll a$, where $\Delta_{m}$ is the mode half-width, $a$ the plasma radius. For the modes with $\Delta_{m} \ll a$ the denominator in equation (21) is proportional to $\Delta_{m}^{-1}\left[(\partial \Phi / \partial r)^{2} \propto \Delta_{m}^{-2}\right.$ and $\left.\int \mathrm{d} r \propto \Delta_{m}\right]$. The numerator considerably depends on the orbit width and the mode width. When the orbit width is negligible, $r=\bar{r}, \Phi_{m, n, l=0}=\Phi_{m, n}, \Phi_{m, n, l \neq 0}=0$, $J_{0}\left(\xi_{m l \mu}\right)=1$, and $J_{q}\left(\xi_{m l \mu}\right)=0$ for $q \neq 0$. Then the numerator is proportional to $n \Delta_{m}^{-1}$ provided that the term proportional to spatial derivative in equation (7) dominates (i.e., when the instability is driven by spatial inhomogeneity of the energetic ions). This leads to the well-known result of the local theory that the growth rate grows linearly with the mode numbers, $\gamma \propto n$. In another limit case, $\Delta_{m} \ll \Delta_{b}$, the numerator of equation (21) decreases with $m$. To see it, we note that, first, in this case $\int \mathrm{d} \bar{r}$ is proportional to $\Delta_{b}$ (rather than to $\Delta_{m}$ ), second, $J_{q}^{2}\left(\xi_{m l \mu}\right) \propto \xi_{m l \mu}^{-1} \propto(m+l+j \mu)^{-1}$, and third, $\Phi_{m, n, l} / \Phi_{m, n} \ll 1$. To be more specific, we take $\Phi_{m, n}(r)=\hat{\Phi}_{m, n} \exp \left[-\left(r-r_{*}\right)^{2} / \Delta_{m}^{2}\right]$. Then we can use features of the $\Phi_{m, n, l}$ harmonics considered in Appendix B. As a result, we conclude that $\gamma \propto \Delta_{m} n / \xi_{m l \mu}$ for $\Delta_{m} / \Delta_{b} \ll 1$. Now we take $n \propto m$ (which is true for $k_{\|} \ll k_{\perp}$ ) and assume that $\Delta_{m} \propto m^{-1}, m \gg \mu, l$. We obtain that the growth rate decreases with the mode numbers as $\gamma \propto m^{-1}$. Note that similar dependence of $\gamma$ on $m$ was obtained for TAE modes in tokamaks in [1], but later it was found that $\gamma \propto m^{-2}[2]$. Probably, the latter result takes place in large-shear systems, in which case the mode structure has the outer mode width, $\Delta_{m}$, and inner mode width, $\Delta_{\text {inner }}$. For low-shear systems,

the mode structure is described by the Gaussian considered above, i.e., it is characterized by the only characteristic width, $\Delta_{m}$. This is shown for the gap modes in Appendix C. In addition, the eigenmode calculated for a particular shot of Wendelstein 7-AS in section 3 can be approximated by a Gaussian with $\Delta_{m}=2.6 r_{*} / m$.

By means of a similar analysis we can evaluate the growth rate of the instability with $\Delta_{b} \gg \Delta_{m}$. We obtain:

$$
\gamma \sim \mathcal{A} \gamma_{0}\left(\frac{\Delta_{m}}{\Delta_{b}}\right)^{2}
$$

where $\gamma_{0}$ is the growth rate calculated in the local approximation neglecting the particle orbit width. In the case when the fast ion population is produced by NBI, the growth rate $\gamma_{0}$ is $[16]$,

$$
\frac{\gamma_{0}}{\omega}=\mathcal{Z} \frac{\pi}{64} \frac{\beta_{b} v_{A}^{2}}{r^{2} \omega^{2}} \sum_{\mu \nu} \mu^{2} \epsilon_{B}^{(\mu \nu)^{2}} Q_{0},
$$


$\mathcal{Z}=2$ when one of the mode components mainly contribute to the mode energy, $\mathcal{Z}=1$ when the mode consists of two dominant components equally contributing to the mode energy, $Q_{0} \equiv Q(\xi=0, l=q=0), Q$ will be defined later [by equations (30)-(32)], the factor $\mathcal{A}>1$ takes into account that different terms in equation (21) can contain the same resonance condition because different $q$ and $l$ can lead to the same resonant velocity. For instance, the zero-orbit-width resonance $l=q=0$ with $j \mu=1$ coincides with the finite-orbit-width resonances $q=l \neq 0$ and $q=l-2$. The estimate (26) is valid when the fast ions with standard orbits mainly contribute to the growth rate.

Let us consider the energetic ions consisting only of the beam particles. Then the energetic ion pressure is anisotropic, $p_{b \|} \gg p_{b \perp}$ (the subscript " $b$ " labels the beam particles). Therefore, we can define $\beta_{b}$ as

$$
\beta_{b}=8 \pi p_{b \|} / B_{0}^{2}=8 \pi M_{b} \int \mathrm{d}^{3} v v_{\|}^{2} F_{0} / B_{0}^{2} .
$$

We assume that $F_{0}=F_{0}(r, v, \chi)$ with $\chi^{2}=1-\lambda \approx\left(v_{\|} / v\right)^{2}$. Then we obtain the growth rate in a form convenient for the practical use as follows [cf. equations (40) and (41) of the mentioned reference]:

$$
\gamma_{b}=\frac{\pi \beta_{b}(0) v_{A}^{2}(0) \sum \int_{\bar{\rho}_{\min }}^{\bar{\rho}_{\max }}(\mathrm{d} \bar{\rho} / \bar{\rho}) G_{m l \mu}^{n \nu j}\left[G_{m^{\prime} l^{\prime} \mu^{\prime}}^{n^{\prime} \nu^{\prime} j}\right]^{*} \mathcal{Q}}{32 a^{2} \omega \sum_{m, n} \int_{0}^{1} \mathrm{~d} \rho \rho\left[\left|\Phi_{m, n}^{\prime}\right|^{2}+\left(m^{2} / \rho^{2}\right)\left|\Phi_{m, n}\right|^{2}\right] n_{i}(\rho) / n_{i}(0)}
$$

where $\rho=r / a, \bar{\rho}=\bar{r} / a, \bar{\rho}_{\min }$ and $\bar{\rho}_{\max }$ are determined by the equations $\bar{\rho}_{\min }=$ $\Delta_{b}\left(w, \bar{\rho}_{\min }\right) / a$ and $\bar{\rho}_{\max }=1-\Delta_{b}\left(w, \bar{\rho}_{\max }\right) / a$, respectively, $\Phi_{m, n}^{\prime}=\partial \Phi_{m, n} / \partial \rho, \kappa_{\rho ; \mu, \nu}=$ $\partial \epsilon_{B}^{(\mu \nu)} / \partial \rho$ for $\mu \neq 0, \nu \neq 0$,

$$
\mathcal{Q}=\frac{|w| \int_{|w|}^{|w| / \sqrt{\epsilon_{\mathrm{eff}}}} \mathrm{d} u J_{q}\left(\xi_{m l \mu}\right) J_{q^{\prime}}\left(\xi_{m l \mu}\right)\left(u^{2}+w^{2}\right)^{2}\left[\frac{\partial}{\partial u}+\frac{\left(1-\chi_{r}^{2}\right)}{u \chi_{r}} \frac{\partial}{\partial \chi_{r}}+u \frac{\omega_{* b}}{\omega}\right] F_{0}\left(\rho, u, \chi_{r}\right)}{2 \int_{0}^{\infty} \mathrm{d} u u^{4} \int_{-1}^{1} \mathrm{~d} \chi \chi^{2} F_{0}(r=0, u, \chi)}
$$

$u=v / v_{0}$ with $v_{0}$ a characteristic velocity of the injected ions, $w=v_{\|}^{\text {res }}(\rho) / v_{0}$, $v_{\|}^{\text {res }}(\rho)$ is defined by equation $(25), \chi_{r}=w / u$ is the resonant pitch angle, $\omega_{* b}=$ $n v_{0}^{2}\left(\omega_{B} \iota r\right)^{-1} \partial \ln \left(F_{0}\right) / \partial r, \epsilon_{\text {eff }}$ is determined from the condition that the particle trapping parameter equals unity. For the parallel neutral injection in plasmas with a large aspect ratio, most energetic ions typically have pitch angles close to unity, in which case $\mathcal{Q}$ does not depend on $\epsilon_{\text {eff }}$.

In many cases the distribution function of the beam ions can be approximated as

$$
F_{0}=\sum_{\sigma=1}^{s} F_{0}^{\sigma}, \quad F_{0}^{\sigma}=\frac{1}{2 \pi \chi_{0}^{2}} \frac{n_{b}^{\sigma}(r)}{v^{3}+v_{c}^{3}} \delta\left(\chi-\chi_{0}\right) \eta\left(v_{\sigma}-v\right),
$$

where $v_{\sigma}$ is the birth velocity, $n_{b}^{\sigma}=p_{b \|}^{\sigma} / \mathcal{E}_{\sigma}, \mathcal{E}_{\sigma}=M_{b} v_{\sigma}^{2} / 2, p_{b \|}^{\sigma}=M_{b} \int \mathrm{d}^{3} v v_{\|}^{2} F_{0}^{\sigma}$ is the partial beam pressure along the magnetic field, $v_{c}=\left(1.33 M_{e} n_{e}^{-1} \sum_{i} n_{i} Z_{i}^{2} / M_{i}\right)^{1 / 3} v_{T e}$ with $v_{T e}=\sqrt{2 T_{e} / M_{e}}, Z_{i}$ is the charge number, $s$ is an integer. Then we obtain for $\left|\chi_{0}\right| \neq 1$ : 


$$
\begin{aligned}
& \mathcal{Q}=\frac{1}{I} \sum_{\sigma} \frac{\eta\left(\chi_{0} u_{\sigma}-w\right) J_{q}\left(\xi_{m l \mu}\right) J_{q^{\prime}}\left(\xi_{m l \mu}\right) w^{2}}{\chi_{0}^{2}\left(1+u_{c}^{3} \chi_{0}^{3} / w^{3}\right)} \\
& \times\left\{w^{2}\left(\frac{1}{\chi_{0}^{2}}+1\right)^{2}\left[\frac{\omega_{* b}}{\omega}-\frac{\left|\chi_{0}\right| \delta\left(w-u_{\sigma} \chi_{0}\right)}{u_{\sigma}}\right]\right. \\
& \left.+\frac{1}{\left(1+u_{c}^{3} \chi_{0}^{3} / w^{3}\right)}\left[\frac{3}{\chi_{0}^{4}}-\frac{2}{\chi_{0}^{2}}-5+2 u_{c}^{3}\left(\frac{3}{\chi_{0}^{2}}+2-\chi_{0}^{2}\right) \frac{\chi_{0}}{w}\right]\right\} \frac{n_{b}^{\sigma}(\rho)}{n_{b}(0)},
\end{aligned}
$$

where $|w|<1, v_{0}=v_{1}, v_{1} \equiv \max \left(v_{\sigma}\right), \chi_{0} w>0, u_{\sigma}=v_{\sigma} / v_{1}, u_{c}=v_{c} / v_{1}, \xi_{m l \mu}$ is taken at $v_{\|}=v_{\|}^{\text {res }}$, i.e., $\xi_{m l \mu}=(m+l+j \mu)\left(R_{0} / r\right) \epsilon_{t}^{\prime} \iota^{-1} r_{B 1} w$ with $r_{B 1}=v_{1} / \omega_{B}, I \equiv$ $\sum_{\sigma=1}^{s} 2 \nu_{\sigma} \int_{0}^{u_{\sigma}} \mathrm{d} u u^{4} /\left(u^{3}+u_{c}^{3}\right)$ with $\nu_{\sigma}=n_{b}^{\sigma}(0) / n_{b}(0), I \approx \sum_{\sigma} \nu_{\sigma} u_{\sigma}$ for $u_{c}^{3} \ll 1$. When $|w|>\left|\chi_{0}\right|$ or $\chi_{0} w<0, \mathcal{Q}=0$ because in this case the resonant particles are absent. The term proportional to $\omega_{b *}$ describes the destabilization of the waves due to the spatial inhomogeneity of the beam ions. The singular term proportional to $\delta\left(w-u_{\sigma} \chi_{0}\right)$ describes the damping caused by sharp drops of $F_{0}$ when $u=u_{\sigma}$; for more realistic distribution function we have to replace $\delta\left(w-u_{\sigma} \chi_{0}\right)$ by $\Delta^{-1}\left(u_{\sigma} \chi\right)=\left(\chi_{0} \Delta u_{\sigma}+u_{\sigma} \Delta \chi_{0}\right)^{-1}$, with $\Delta \chi$ the pitch-angle spread of the distribution around $\chi_{0}$ and $\Delta u_{\sigma}$ the energy spread at $u=u_{\sigma}$. The rest of the terms arises from the competition between the destabilizing influence of the velocity anisotropy and the stabilizing effect of the monotonically decreasing $F_{0}(v)$; these terms are destabilizing for $w<\left|\chi_{0}\right|<\chi_{\max }$, with $\chi_{\max }=0.77$ for $u_{c} / w \ll 1$ and $\chi_{\max } \rightarrow 1$ (but $\chi_{\max } \neq 1$ ) for $u_{c}^{3} / w^{3}>0.5$.

For the special case of $\chi_{0}=1$ we get:

$$
\begin{aligned}
& \mathcal{Q}=\frac{4}{I} \sum_{\sigma} \eta\left(u_{\sigma}-|w|\right) J_{q}\left(\xi_{m l \mu}\right) J_{q^{\prime}}\left(\xi_{m l \mu}\right) \frac{w^{2}}{1+u_{c}^{3} / w^{3}} \\
& \times\left\{w^{2}\left[\frac{\omega_{* b}}{\omega}-\frac{\left|\chi_{0}\right| \delta\left(w-u_{\sigma} \chi_{0}\right)}{u_{\sigma}}\right]-\frac{2-u_{c}^{3} / w^{3}}{1+u_{c}^{3} / w^{3}}\right\} \frac{n_{b}^{\sigma}(\rho)}{n_{b}(0)} .
\end{aligned}
$$

The analysis above is based on the assumption that the energetic ions have standard orbits, i.e., they encircle the magnetic axis. However, when the modes are localized in the core, the particles that do not encircle the axis (i.e., that are passing in the toroidal direction but trapped in the poloidal angle) may play an important role. The orbits of such semi-trapped particles are approximately circles in the poloidal cross section (like the orbits of standard circulating particles), but all these circles are centered at $r=\Delta_{b}, \vartheta=0$ or $\vartheta=\pi$ (depending on the sign of $v_{\|}$), and the radius of the circles varies from zero to $\Delta_{b}$. Taking into account features of semi-trapped particles (described in Appendix A), we modify equation (29) for the growth rate in order to describe the contribution of the mentioned particles. This can be done as follows. First, the integral in equation (29) should be taken over the region $0<\bar{\rho}<\bar{\rho}_{\text {min }}$. Second, the Bessel function argument, $\xi_{m, l, \mu}$, should be replaced by $\xi_{m, \mu}^{0} \equiv(m+j \mu) \bar{r} /\left|\Delta_{b}\right|$. Third, $\Phi_{m, n, l}$ should be replaced by $\Phi_{m, n, l}^{0}$ defined by equation (B.5). Fourth, the resonance condition should be written as

$$
\omega=\left[(l-q) \iota-n_{0}-j \nu N\right] \frac{v_{\|}^{\mathrm{res}}}{R_{0}} .
$$


We observe that the poloidal mode number $(m)$ and the poloidal number of the equilibrium magnetic field $(\mu)$ are absent in equation (34). This is a consequence of the fact that the particles that are well trapped poloidally do not "feel" the poloidal structures of the mode and $B_{0}$. The mentioned feature of the resonance of the poloidally localized particles was first noticed in [19]. The toroidal mode number $(n)$ and the toroidal number of the equilibrium magnetic field are present in equation (34) because the considered particles are circulating in the toroidal direction. Note that although the obtained equation for growth rate is valid, strictly speaking, only for the particles well-localized poloidally $\left(\bar{r} \ll \Delta_{b}\right)$, one can expect that it will reasonably approximate the growth rate produced by particles with $\bar{r} \lesssim \Delta_{b}$.

\section{Modelling Alfvén instability in the W7-AS shot \#34723}

\subsection{Experimental observations}

In order to demonstrate effects of the finite orbit width, below we consider an Alfvén instability observed in the W7-AS shot \#34723. This instability had a bursting character and was characterized by strong frequency chirping down, from about $70 \mathrm{kHz}$ to $45 \mathrm{kHz}$, as shown in figure 1. The instability was strongest at the final stage of the bursts, when thermal crashes occurred. The duration of the instability bursts was about $2.5 \mathrm{~ms}$, the repetition period of the bursts was 8-10 ms. The plasma energy grew with time on average. Eventually, the bursts stopped, and only relatively weak steady-state Alfvénic activity with a frequency equal to that at the beginning of the bursts remained, without a visible influence on the plasma at this stage. The poloidal mode numbers, observed by Mirnov coils during the instability, were $\left|m_{1}\right|=3$ and $\left|m_{2}\right|=5$ with a weak $\left|m_{3}\right|=4$ component. The toroidal mode numbers were not detected. The maximum amplitude of the field perturbations at the plasma boundary was $\widetilde{B}_{\theta} / B \approx 10^{-4}$. The final stage of each burst of the instability was accompanied by a rather strong drop of the plasma energy, up to $30 \%$. However, no noticeable effect on the plasma density was observed. Soft Xray measurements showed oscillations with the instability frequency in the core but not at the periphery. Inversion of the sawtooth-like oscillations of the electron temperature occurred at $r / a \sim 2 / 3$. Plasma was rather dense, $n_{e}(0)=10^{14} \mathrm{~cm}^{-3}$, with a very flat density profile, and relatively cold, the central temperature was $T(0)=290 \mathrm{eV}$. The magnetic field was $B=1.21 \mathrm{~T}$. The instability was caused by injected protons, the energies of the injected particles were $48 \mathrm{keV}, 24 \mathrm{keV}$, and $16 \mathrm{keV}$. The injection was balanced, i.e., there were particles with $v_{\|}>0$ and $v_{\|}<0$. These particles were injected into deuterium plasma. The NBI deposition profile was rather peaked (figure 2).

Before proceeding to analysis of these experimental observations, we should note that we will make a number of plausible conjectures because some important experimental data are not available (e.g., the toroidal mode number of the instability, the exact fraction of hydrogen in the deuterium plasma etc.). Therefore, we cannot rule out a possibility of other interpretations of the observed phenomena than that proposed 
in this work.

\subsection{Equilibrium analysis}

We begin our analysis of the experimental observations of Alfvénic activity in the shot \#34723 with the calculation of the Fourier spectrum of the magnetic field and metric tensor coefficients in Boozer coordinates [20]. With this aim, we used the equilibrium code VMEC [21] to calculate the equilibrium configuration and the code Mapping [22] to transform the VMEC results to Boozer coordinates (in addition to a mapping from Boozer coordinates to cylindrical ones, Mapping calculates three metric tensor components, which are sufficient to find the rest of the components [23]). However, the results of the calculations turned out to be unsatisfactory for the central region of the plasma. Analytical expressions for the metric tensor at the magnetic axis [7] demand that the metric tensor components should satisfy certain relationships, which were broken with the error of $30-50 \%$ at the magnetic axis. The reason for this inconsistency was that the output of VMEC is not accurate in the core region of the plasma, which occupies typically about $1 / 4$ of the plasma radius. This inaccuracy was "inherited" by the metric tensor calculated by Mapping. To resolve this problem, we wrote a new computer program on the basis of the metrics-processing part of the code COBRA [7]. The program approximates the Fourier harmonics of the metric tensor components given by Mapping by low-order polynomials, which take into account the asymptotic properties of the harmonics at the magnetic axis. During the approximation, the data in the core region, where VMEC gives largest errors, are ignored. After that, the components are recovered from the polynomials. As a result, they are smoothed, and the data for the core region are replaced by new extrapolated data. Only after that, the rest of the components are calculated. This procedure has enabled us to reduce drastically the inconsistency between the metric tensor components (e.g., to about $7 \%$ for the analysed shot \# 34723). The results are shown in figure 3.

The $\iota$ profile obtained from equilibrium calculations was flat with $\iota(a)=0.405$, $\iota_{\max }=0.425$; however, neither the bootstrap current nor the beam-induced current (the latter does not vanish even during balanced injection due to the finite width of the beam ion orbits) were taken into account, which means that the central rotation transform may slightly differ from the mentioned value. Therefore, in addition, we considered several other similar profiles of $\iota(r)$. The calculated rotational transform as well as the corrected ones (to satisfy the conditions described below) are shown in figure 4 .

Because protons were injected into a deuterium plasma, the plasma consisted of a mixture of deuterium and hydrogen. This leads to an uncertainty in the magnitude of the Alfvén velocity and, thus, in the frequencies of Alfvén modes. Therefore, we need to evaluate the fraction of hydrogen in the plasma. First of all, we write an equation for the total number of injected and confined particles, $I_{\Sigma}$, as follows:

$$
I_{\Sigma}=\frac{\left(1-f_{\text {loss }}\right) P_{\text {inj }}}{\mathcal{E}_{1} I_{1} / I_{\Sigma}+\mathcal{E}_{2} I_{2} / I_{\Sigma}+\mathcal{E}_{3} I_{3} / I_{\Sigma}}
$$


where $P_{\text {inj }}=970 \mathrm{~kW}$ is the injected power, $f_{\text {loss }}$ is the fraction of the lost injected particles; $I_{1} / I_{\Sigma}=0.2, I_{2} / I_{\Sigma}=0.32, I_{3} / I_{\Sigma}=0.48$ are the fractions of the particles injected with the energies $\mathcal{E}_{1}=48 \mathrm{keV}, \mathcal{E}_{2}=24 \mathrm{keV}$, and $\mathcal{E}_{3}=16 \mathrm{keV}$, respectively. Assuming that $f_{\text {loss }}=20 \%$, the plasma radius $a=15 \mathrm{~cm}$, and $R_{0}=200 \mathrm{~cm}$, we obtain $S \equiv I_{\Sigma} / V_{p l}=2.2 \times 10^{14} \mathrm{~s}^{-1} \cdot \mathrm{cm}^{-3}$. Taking into account that hydrogen was accumulated for $\Delta t \sim 0.1 \mathrm{~s}$ before the moment of the observation, we obtain the average hydrogen particle density as $\bar{n}_{H}=2.2 \times 10^{13} \mathrm{~cm}^{-3}$. As we have already mentioned, the radial profile of the beam ions was rather peaked. On the other hand, the particle confinement time in W7-AS is typically very large, $\tau_{n} \gg 0.1 \mathrm{~s}$, which implies that the diffusion weakly affects the radial profile of protons in the plasma core, and the radial profile of the thermalized particles is similar to that of the beam particles. Taking this into account, we conclude that the $\mathrm{H}$-ion density in the near-axis region exceeds $\bar{n}_{H}$ by a factor of three $\left[\right.$ for $\left.n_{b}=n_{b}(0)\left(1-r^{2} / a^{2}\right)^{2}\right]$. Therefore, below we assume $n_{H} /\left.n_{e}\right|_{r=0}=60 \%$.

Now we can calculate the Alfvén continuum. This is done with the code COBRA [7]. Using the rotational transform calculated with the code NEMEC [24] $\left(\iota_{*} \equiv \max \iota(r)=\right.$ 0.427 , see figure 4 , curve a), we obtain the picture shown in figure $5(\mathrm{a})$. This AC is characterized by a wide ellipticity-induced gap in the frequency range that includes the experimentally observed frequencies, which, on the first sight, indicates that the observed instability with the poloidal mode numbers $m=3$ and $m=5$ was associated with Ellipticity-induced Alfvén Eigenmodes (EAE). However, a more detailed analysis below does not support this conclusion. This analysis is based on the fact that a necessary condition for the existence of EAE modes is that two cylindrical Alfvén branches, $\omega_{1}=|m \iota(r)-n| v_{A}(r) / R_{0}$ and $\omega_{2}=|(m+2) \iota(r)-n| v_{A}(r) / R_{0}$ intersect at a certain radius. For the observed poloidal mode numbers $|m|=3$ and $|m|=5$ this occurs when $\iota=|n| / 4$, i.e., when $\iota=0.25$ for $|n|=1, \iota=0.5$ for $|n|=2, \iota=0.75$ for $n=3$ etc., whereas in our calculations $0.405<\iota(r)<0.427$. Therefore, the observed instability cannot be a conventional EAE mode. Instead, it can be either a non-EAE gap mode or a continuum mode with two dominant harmonics coupled by the ellipticity Fourier harmonics of the magnetic field and metric tensor. The eigenmode calculation with the code BOA-E [10] with the use of the two-harmonic approximation has shown that there are discrete eigenmodes with $|m|=3$ and $|m|=5$ for $\iota_{*}=0.427$. However, their frequencies are close to the top of the continuum branch with $|m|=5,|n|=2$, i.e., their frequencies are much less than the experimentally observed frequencies. Furthermore, coupling with other Fourier harmonics shifts down the mentioned branch, so that the eigenmode frequencies are actually only about $20 \mathrm{kHz}$ (the $|m|=5,|n|=2$ continuum branch shown by red in figure 5 is located below $20 \mathrm{kHz}$ ). On the other hand, the curve corresponding to the AC branch with $|m|=3,|n|=1$ is characterized by frequencies about $40 \mathrm{kHz}$ (the magenta line in figure $5(\mathrm{a})$ ). However, we failed to find discrete eigenmodes with $|n|=1$. Furthermore, the $(3,1)$ branch is coupled with the $(1,1)$ branch much stronger than with the $(5,1)$ branch [the $(5,1)$ branch is located in a very high frequency range]. Because of this, if the $(3,1)$ harmonic were destabilized, the $(1,1)$ harmonic, rather than the $(5,1)$ harmonic, would be detected by Mirnov coils (in 
contrast to the experiment). We conclude from here that only modes with $n=2$ can be destabilized. If so, it seems that the only possibility to increase the calculated frequency is to modify $\iota(r)$.

Let us take $\iota(r)$ presented by the upper curve in figure 4 , which is within the error bar. The calculated AC is shown in figure $5(\mathrm{~b})$. The $(5,2)$ curve is higher in this case, so that one can expect the presence of discrete AEs at $\sim 50 \mathrm{kHz}$. Calculations with BOA-E confirmed this possibility. The calculated eigenmode frequencies lie close to a maximum (lower frequency) and a minimum (higher frequency) of the corresponding branches of AC. Note that taking larger $\iota_{*}$, we would obtain the $(5,2)$ branch with a higher frequency. However, larger $\iota_{*}$ is difficult to reconcile with the rotational transform calculated by NEMEC. Therefore, it is reasonable to assume that better agreement between the calculated mode frequencies and experimentally observed frequencies could be reached if we take into account the Doppler frequency shift arising because of the poloidal plasma rotation. For instance, the poloidal rotation with the velocity $v_{\text {pol }} \sim 0.6 \mathrm{~km} \cdot \mathrm{s}^{-1}$ at $r \sim 3 \mathrm{~cm}$ produces the Doppler shift $\Delta \omega_{\text {dop }}=m v_{\text {pol }} / r \sim 15 \mathrm{kHz}$ for a mode with $m=5$, which provides agreement between the calculated eigenfrequency and the frequency observed at the beginning of the instability burst. Experimental data indicate that a much larger velocity of the poloidal rotation of impurity ions, up to $10-30 \mathrm{~km} \cdot \mathrm{s}^{-1}$, in the region of $r=9-15 \mathrm{~cm}$, took place in W7-AS [17]. Unfortunately, experimental data on the poloidal rotation of the bulk plasma are absent. On the other hand, according to our recent publication, the Alfvén-induced heat transport is accompanied by the formation of sheared poloidal rotation [14]. Note that the toroidal rotation could hardly considerably contribute to $\Delta \omega_{\text {dop }}$ because the aspect ratio of the torus was large and the expected velocity of the toroidal rotation was relatively low (toroidal velocities higher than $5-10 \mathrm{~km} \cdot \mathrm{s}^{-1}$ were never observed in W7-AS shots with balanced NBI).

The mode frequency strongly depends on $\iota_{*}$. Therefore, a small change of $\iota$ considerably changes the mode frequency. In order to see this, we assume that $v_{A}(r) \approx$ const in the region of the instability (the plasma density profile is very flat) and that the maximum of the rotational transform evolves from $\iota_{1}$ to $\iota_{2}$. Then, approximating the mode frequency as $\omega=C k_{\|}\left(\iota_{*}\right) v_{A}$ with $C=$ const, we obtain the corresponding change of the mode frequency:

$$
\frac{\omega_{2}-\omega_{1}}{\omega_{1}}=\frac{\iota_{2}-\iota_{1}}{\iota_{1}-n / m} \approx-0.3
$$

for $m / n=5 / 2, \iota_{1} \equiv \iota_{*}\left(t_{1}\right)=0.47$, and $\iota_{2} \equiv \iota_{*}\left(t_{2}\right)=0.45$. Equation (36) is in reasonable agreement with (slightly overestimates) the frequency change obtained from AC calculations with COBRA [7].

Taking into account equation (36) and the above analysis, we assume that the rotational transform at the initial and final stages of the instability is described by curves c and b, respectively, in figure 4 .

The above consideration suggests the following explanation for the observed frequency chirping: The instability influences the rotational transform, reducing its maximum magnitude. A possible mechanism leading to the change of the rotational 
transform is the instability-induced redistribution of beam ions with a certain sign of $v_{\|}$, as determined by the resonance condition given by equation (25) (an analysis of the resonance will be carried out in the next subsection). The current $\delta j$ generated as a result of such a redistribution can be evaluated as follows. We assume for simplicity that only particles with the injection energy $\mathcal{E}_{1}=48 \mathrm{keV}$ are redistributed. Then

$$
|\delta j|=e_{b} n_{b 1} v_{b 1}\left(1-z_{\text {eff }}^{-1}\right) \frac{\left|\delta n_{b 1}\right|}{n_{b 1}},
$$

where the subscript " 1 " refers to the beams with the injection energy $\mathcal{E}_{1}, z_{\text {eff }}$ is the effective charge number, $\delta n_{b 1} \equiv n_{b 1}^{+}-n_{b 1}^{-}, n_{b 1} \equiv n_{b 1}^{+}+n_{b 1}^{-}, n_{b 1}^{+}$and $n_{b 1}^{-}$are the densities of co- and counter-injected particles, respectively, $n_{b 1}^{+}=n_{b 1}^{-}$before the instability bursts. For the parameters used above in this section and $z_{\text {eff }}=3$ we obtain $\left|\delta j_{b}\right|=10.37\left|\delta n_{b 1}\right| / n_{b 1} \mathrm{~A} \cdot \mathrm{cm}^{-2}$. On the other hand, the generated current changes the rotational transform, $\delta \iota=2 \pi R \delta j /(B c)$, which is true for the near-axis region. We obtain from here that in order to provide $\delta \iota=0.02$, as required for the frequency chirping of $30 \%$, we need $\delta j=1.94 \mathrm{~A} \cdot \mathrm{cm}^{-2}$. Now, using equation (37), we have $\delta n_{b 1} / n_{b 1}=0.187$. This implies that $\beta_{b}$ and, thus, the instability drive are weakly affected. To see it, we take into account that $\beta_{b}=2.6 \beta_{b 1}$ [this estimate is made for the parameters given after equation (35)]. Then we find the following change of $\beta_{b}: \delta \beta_{b} / \beta_{b}=7.2 \%$. The change of $\beta$ associated with the current generation is even smaller when particles with the birth energies $\mathcal{E}_{2}$ and $\mathcal{E}_{3}$ are redistributed.

The frequency chirping is rather fast, the time derivative decreasing at the final stage of the instability burst. At the initial stage, the frequency drops from about 70 $\mathrm{kHz}$ to $50 \mathrm{kHz}$ for $0.7 \mathrm{~ms}$, as shown in figure 1 . To see whether this is consistent with our model, we solve the following model equation:

$$
\frac{\partial \iota}{\partial t}=\frac{1}{r} \frac{\partial}{\partial r} \frac{D_{\iota}}{r} \frac{\partial}{\partial r} r^{2}\left(\iota-\iota_{\infty}\right)
$$

where $D_{\iota}=c^{2} /\left(4 \pi \sigma_{\|}\right), \sigma_{\|}$is the electrical conductivity along the magnetic field, $\iota_{\infty}=\iota_{\infty}(r)$ is the rotational transform in the infinite time after the burst. The result is shown in figure 6 . We observe that the rate of change of the central iota is largest at the initial stage, which agrees with the experiment. Moreover, iota changes by 0.02 for $0.7 \mathrm{~ms}$, again in agreement with the experiment.

These estimates demonstrate that the evolution of the plasma equilibrium can be responsible for the frequency chirping. However, they do not exclude that other factors (in addition to the change of the rotational transform) may be of importance.

It follows from our analysis that the observed mode is not a gap mode; therefore, it can be identified as an non-conventional Global Alfvén Eigenmode (NGAE) with frequency lying above the AC. The eigenmode for the final stage of the instability burst is shown in figure 7. This eigenmode is core localized. As shown in [14], it generates a Kinetic Alfvén Wave (KAW), which extends the region affected by the instability up to $r / a \sim 2 / 3$. The KAW causes anomalous electron thermal conductivity at the final stage of each instability burst, which accounts for the thermal crashes observed in the 
experiment. In addition, the extension of the instability region by the KAW facilitates detecting the instability by the Mirnov coils.

Because the eigenmode shown in figure 7 is localized close to the magnetic axis, and, in addition, the beam radial profile was peaked, one can expect that particles with non-standard orbits (semi-trapped particles) will play a considerable role in the destabilization of the mode. Typical orbits of injected ions are shown in figure 8.

Note that the evaluated $\beta_{b}$ is considerably smaller (by a factor of 10) than $\beta_{p}$ $\left(\beta_{p}=8 \pi p / B^{2}\right.$, where $p$ is the plasma pressure). This indicates that the fast-ion-induced instabilities can be treated perturbatively. Below we follow this approach.

\subsection{Destabilization of Alfvén eigenmodes}

The AE modes are destabilized by the resonant interaction with beam ions. The corresponding resonances in the plasma frame are given by equations (25) and (34). One can see that in the considered case of low-frequency oscillations and small $\iota$, only axisymmetric resonances $(\nu=0)$ are of importance. The matter is that a large magnitude of the field periods $(N=5)$ and small $\iota$ make the $\nu \neq 0$ resonances important only for high-frequency modes (e.g., for the helicity-induced AEs); for the modes with $\omega \ll N v_{A} / R_{0}$, the $\nu \neq 0$ resonances lead to low resonant velocities. Because the toroidal harmonic is dominant among the harmonics of $B$ (and the curvature) with $\nu=0$, we consider the toroidicity-induced resonances $(\mu=1)$.

We begin with a simple analysis. Neglecting the orbit width and assuming that the fast ions have standard orbits, we have a "sideband" resonance:

$$
w_{1}=\frac{\omega R_{0}}{v_{1}(2-4 \iota)}
$$

which provides interaction with both components $\left(m_{1}=-5\right.$ and $\left.m_{2}=-3\right)$ of the mode. For the W7-AS parameters $\left(R_{0}=200 \mathrm{~cm}, v_{A}=2 \times 10^{8} \mathrm{~cm} \cdot \mathrm{s}^{-1}, v_{1}=3 \times 10^{8} \mathrm{~cm} \cdot \mathrm{s}^{-1}\right)$ it can be written as $(2-4 \iota) w=0.167 \omega_{40}$, where $\omega_{40}$ is $\omega$ normalized to $40 \mathrm{kHz}$. In particular, for $\iota=0.45$ we have $\omega_{40}=1.2 w$. We conclude from here that the resonance given by equation (39) involves most energetic particles, $0.83<w<1$, when $40 \mathrm{kHz}<\omega<48 \mathrm{kHz}$, i.e., at the final stage of the instability bursts. However, this resonance cannot provide wave-particle interaction at the initial stage of the bursts, which was characterized by frequencies $50-70 \mathrm{kHz}$ (because the resonant particles with $w>1$ are absent). Therefore, we consider an additional resonance, $\omega=k_{\|} v_{\|}$, which we will refer to as the "basic" resonance. This resonance can be obtained from equation (25) by taking, e.g., $j \mu=q, l=\nu=0, m=-5, n=-2$. It leads to the resonant velocities determined by

$$
w_{2}=-\frac{\omega R_{0}}{v_{1}(5 \iota-2)} \quad \text { and } \quad w_{3}=\frac{\omega R_{0}}{v_{1}(2-3 \iota)}
$$

for the components with $m_{1}=-5$ and $m_{2}=-3$, respectively. Equations (39) and (40) yield the dependencies of $w$ on $\omega$ for various $\iota$ shown in figure 9. However, not 
all these resonant velocities take place in reality. The matter is that, according to subsection 3.2, the mode frequency depends on $\iota$. For the considered mode we can use the approximation $\omega=\left|5 \iota_{*}-2\right| v_{A} / R_{0}$. This leads to the resonant velocities determined by the resonances mentioned above and the mode frequencies shown in figure 10 . We observe that there is the only important resonance at high $\iota$ (i.e., in the high frequency region, $\omega \gtrsim 40 \mathrm{kHz}$ ). This is the basic resonance involving particles with $v_{\|}<0$, $v_{\|}^{\text {res }}\left(\iota_{*}\right) \approx$ const. The situation in the low frequency region, $\omega \lesssim 40 \mathrm{kHz}$ is different: here there is, in addition, a sideband resonance involving particles with $v_{\|}>0$, $v_{\|}^{\text {res }}$ being largest at $\omega \sim 40 \mathrm{kHz}$ (when it exceeds $\left|v_{\|}^{\text {res }}\right|$ of the basic resonance) and decreasing at smaller frequencies.

We conclude that the decrease of $\iota$ accompanied by the frequency chirping down (the initial stage of the instability bursts) will monotonically increase the growth rate $\left[\gamma \propto \omega^{-2}\right.$, according to equations (27) and (32)] until the moment when $\omega$ falls below $45 \mathrm{kHz}$ : then the sideband resonance will be switched on, which will sharply enhance the instability.

We should emphasise that, in contrast to the sideband resonance, the basic resonance can be produced by the toroidal Fourier harmonic of $B$ only due to diamagnetic component of the curvature (this component is small and, furthermore, it contributes to $\gamma$ due to the finite mode width) or due to the finite orbit width. The latter mechanism seems most important.

Of course, in reality there are many resonances that contribute to the growth rate. Moreover, because $r_{*}<\Delta_{b}$, semi-trapped particles are involved in addition to particles with standard orbits, which complicates the picture. Nevertheless, it is possible to evaluate the growth rate, at least, in the case when there is a resonance with $w \lesssim 1$.

With this purpose, we approximate the beam radial distribution as $n_{b}=n_{b 0}(1-$ $\left.r^{2} / a^{2}\right)^{2}$ and take $\beta_{b}=\beta_{b}^{+}+\beta_{b}^{-}\left[\beta_{b}\right.$ is defined by equation (28), the superscripts "+" and "_" are relevant to co- and counter-injected particles, respectively], $\beta_{b}^{+}=\beta_{b}^{-}=3 \times 10^{-3}$, $\nu_{1}=0.2$. Then, using equation $(27)$, we obtain $\gamma_{0}^{s / b} /\left.\omega\right|_{40 \mathrm{kHz}} \sim 0.5$ for $w=0.8-0.9$, where the superscript "s/b" refers to the sideband resonance. This means that the instability could hardly be studied perturbatively if the orbit width were vanishing. However, in reality $\Delta_{b} \sim 3 \mathrm{~cm}$ for $w=0.8-0.9$, whereas $\Delta_{m} \sim 1 \mathrm{~cm}$; in addition, small $r_{*}\left(r_{*}<\Delta_{b}\right)$ decreases the growth rate by affecting $\Phi_{m, n, l}$. Using equation (26), we obtain $\gamma^{\mathrm{s} / \mathrm{b}} / \omega \sim 0.1 \delta$ with $\delta \sim 1 / 3$ (the $l=0$ Fourier harmonic mainly contributes to the growth rate because $\int\left|\Phi_{m, n, l}\right|^{2} \mathrm{~d} \bar{r}$ is largest for $l=0$; therefore, there are, at least, two important resonances characterized by $q=0$ and $q=-2$, i.e., $\mathcal{A}=2$; the factor $1 / 3$ takes into account that $r_{*}<\Delta_{b}$ ). The growth rate of the instability resulting from the basic resonance, $\gamma^{\text {basic }}$, is $\gamma^{\text {basic }} / \gamma^{\mathrm{s} / \mathrm{b}} \sim\left(w^{\text {basic }} / w^{\mathrm{s} / \mathrm{b}}\right)^{2} \mathcal{A}^{\text {basic }} / \mathcal{A}^{s / b} \sim 0.6, \mathcal{A}^{\text {basic }}=2$, which corresponds to the $q= \pm 1$ resonances. Then the growth rate at the end of the instability burst can be evaluated as $\gamma=\gamma^{\mathrm{s} / \mathrm{b}}+\gamma^{\text {basic }} \sim 1.6 \gamma^{\mathrm{s} / \mathrm{b}}$ and, thus, $\gamma / \omega \sim 0.05$. Below we will show that this estimate a little underestimates the growth rate. To evaluate the growth rate at the beginning of the instability bursts is more difficult because many resonances approximately equally contribute when $w$ is small. 
To calculate the growth rate numerically we use the code GAMMA $[13,18]$ and a new code based on equations (29) and (32). We will refer to the latter code as GAMMA-O (where "O" indicates that finite Orbit width is taken into account).

We begin with calculations neglecting the orbit width. The obtained results are shown in figure 11(a). As expected, the counter-injected beam, $v_{\|}^{\text {beam }}<0$, interacting with the mode through the basic resonance, according to figure 10, does not affect the mode in the used approximation (both the orbit width and the diamagnetic curvature are neglected). In contrast to this, the co-injected beam leads to a strong instability. We observe three peaks. These peaks are a consequence of the fact that the injected particles had the energies of $48 \mathrm{keV}, 24 \mathrm{keV}$, and $16 \mathrm{keV}$. However, in the region of interest, $\omega \gtrsim 40 \mathrm{kHz}$, only particles with the energy above $24 \mathrm{keV}$ contribute to the growth rate. The frequency region associated with the particles with $\mathcal{E} \lesssim 48 \mathrm{keV}$ is rather narrow, which explains why $\gamma \rightarrow 0$ for $\omega>42 \mathrm{kHz}$.

Taking the finite orbit width into account strongly changes this picture, see figure 11(b). First, an instability at high frequencies $(\omega>42 \mathrm{kHz})$ appears due to both counter-injected and co-injected ions. Second, the instability at $\omega \sim 40 \mathrm{kHz}$ is weakened in spite of the fact that a contribution of counter-injected ions is added. The calculated growth rates of the instability are $\gamma_{1} / \omega=0.019$ at $\omega=60 \mathrm{kHz}$ and $\gamma_{2} / \omega=0.054$ at $\omega=40 \mathrm{kHz}$. Thus, we have agreement between the numerical results (for $\gamma_{2}$ ) and the above estimate made on the basis of a qualitative analysis. The growth rate at $40 \mathrm{kHz}$ is less than the growth rate obtained in the zero-orbit-width approximation by a factor of 6.6. Note that the effect of finite orbits at $\omega=42 \mathrm{kHz}$, where $\left.\gamma\right|_{\Delta_{b}=0}$ is maximum, is even more pronounced: $\left.\gamma\right|_{\Delta_{b}=0} / \gamma=8.1$. The destabilization of the Alfvén modes occurs mainly due to fast ions with standard orbits, although the semi-trapped particles contribute quite noticeably (26\% at $60 \mathrm{kHz}$ and $28 \%$ at $40 \mathrm{kHz}$ ).

Figure 12 shows partial growth rates, i.e., the growth rates associated with various individual resonances. It follows from figure $12(\mathrm{a})$ that the resonances with $(l, q)=(0,1),(0,4),(-2,2),(0,0),(-1,-1),(0,-1),(0,-2),(1,1)$ and $(-1,-3)$ play the main role at the final stage of the instability, whereas the resonances with $(l, q)=(0,-1),(0,1),(0,3),(0,-3),(-1,2),(1,0)$ play the main role at the initial stage of the instability, see figure $12(\mathrm{~b})$, the order of these resonances corresponding to their contributions to the growth rate. In particular, the semi-trapped particles contribute mainly due to the sideband resonance with $(l, q)=(0,4),(-2,2)$. The basic resonance with $(l, q)=(0,1)$ dominates for circulating particles with $\chi_{0}<0$. On the other hand, there are several important resonances for circulating particles with $\chi_{0}>0$.

Note that the sideband resonance involves particles with the other sign of $v_{\|}$than the basic resonance does (see figure 10). Therefore, one can expect that the decrease of $\iota$ will be stopped at the stage when the sideband resonance begins to work and, moreover, $\iota$ may start to grow. This may explain why the frequency chirping down stopped after the mode frequency reached the minimum value of about $45 \mathrm{kHz}$ and even a slight increase of $\omega$ was observed at the end of the instability burst. 


\section{Summary and conclusions}

We have developed a theory of Alfvén instabilities driven by circulating and semitrapped energetic ions with finite orbit width. As a result, a general expression for the instability growth rate is obtained, from which it follows that the large orbit width typically decreases the growth rate by affecting the phase of the resonant wave-particle interaction and producing the resonances in the low-energy region. On the other hand, it is concluded that in some cases finite orbits can lead to new instabilities caused by additional high-energy resonant particles. The developed theory is applicable to both low-frequency Alfvén modes (like TAE modes) and high-frequency modes (helicityinduced Alfvén eigenmodes and mirror-induced Alfvén eigenmodes). The theory is also applicable to instabilities associated with other discrete Alfvén eigenmodes possible in stellarators.

The obtained expression for the growth rate includes the contributions of both the particles that encircle the magnetic axis during their orbital motion and the particles that do not encircle the axis (i.e., the particles that are circulating in the toroidal direction but trapped in the poloidal direction). The latter group of the particles may constitute a considerable fraction of injected ions and contribute to the destabilization of core-localized Alfvén modes. A peculiarity of the particles well localized poloidally is that their resonance interaction with the waves almost does not depend on the poloidal mode number and the poloidal inhomogeneity of the equilibrium magnetic field [19].

The code GAMMA-O calculating the instability growth rate with taking the finite orbit width into account is developed. The code extends the code GAMMA. In addition to the total growth rate, it gives a possibility to output the partial growth rates, i.e., contributions associated with individual resonances (characterized by specific $l$, $q$, etc.), individual signs of the longitudinal velocity of the fast ions, individual components of the mode.

It is found that the instability growth rate decreases with the mode numbers as $\gamma \propto m^{-1}$ when the orbit width well exceeds the mode width. This is different from the dependence $\gamma \propto m^{-2}$ obtained for the TAE instability in tokamaks [2], which possibly can be explained by different radial structures of the modes in tokamaks [where the magnetic shear is large $(\hat{s} \sim 1)]$ and low-shear systems $(\hat{s} \ll 1)$ considered here. As we have shown, the radial profile of well-localized Alfvén modes can be approximated by a Gaussian with the half-width $\Delta_{m} \sim r / m$ (in contrast to the structure of well-localized TAE modes in tokamaks) for the gap modes and for the GAE mode in the considered W7-AS shot. On the other hand, $\gamma \propto n$ when the orbit width is small, $\Delta_{b} \ll \Delta_{m}$. Thus, the drive is maximum when $m \lesssim r / \Delta_{b}$. This fact can be used for analysis of experiments where the mode numbers cannot be inferred from experimental data. They can also be used for predictive calculations.

We applied the developed theory to the Wendelstein 7-AS shot \#34723, where a bursting Alfvén instability with the frequency chirping down was observed. Our analysis has shown that this instability can be identified as an NGAE mode (a non- 
conventional GAE mode with a frequency above the Alfvén continuum) having the dominant mode numbers $\left|m_{1}\right|=5,\left|m_{2}\right|=3$ and $|n|=2$. The calculated mode has the width less than the orbit width of the injected ions. Therefore, finite orbits considerably affect the instability: first, they strongly decrease the growth rate at the final stage of each instability burst, which justifies the perturbative approach we have used $\left(\gamma /\left.\omega\right|_{\Delta_{b}=0} \approx 0.4\right)$; second, they trigger the instability at $\omega \sim 60-70 \mathrm{kHz}$ by providing the toroidicity-induced resonant interaction between the energetic ions and the mode. In addition, it is shown that the frequency chirping during the instability bursts may be caused by a small local change of the rotational transform due to a redistribution of beam ions.

An invariant of the motion of well-circulating particles and semi-trapped particles in stellarators with a large number of the field periods $(N \gg 1)$ is derived. This invariant was used to find the orbits of the fast ions destabilizing Alfvén waves.

\section{Acknowledgments}

The work is carried out within the Partner Project Agreement No. P-034e between the Science and Technology Center in Ukraine, the Kyiv Institute for Nuclear Research, and Max-Planck-Institut für Plasmaphysik.

\section{Appendix A. An invariant of motion and the orbits of the well-circulating particles and semi-trapped particles}

We proceed from the following equations of the particle motion:

$$
\dot{\psi}=-\frac{v_{\|}^{2}}{\omega_{B}} \frac{\partial B}{\partial \vartheta}, \quad \dot{\vartheta}=v_{\|} \iota b^{\varphi}+\frac{v^{2}}{r \omega_{B}} \frac{1}{B} \frac{\partial B}{\partial r}, \quad \dot{\varphi}=v_{\|} b^{\varphi},
$$

where the superscripts " $\psi$ ", " $\vartheta$ ", and " $\varphi$ " refer to the corresponding contravariant vector components. It follows from equation (A.1) that

$$
\frac{\mathrm{d} \psi}{\mathrm{d} \varphi}=-\frac{B v_{\|}}{\omega_{B} B^{\varphi}} \frac{\partial B}{\partial \vartheta}
$$

For the orbits located sufficiently far from the magnetic axis, $\Delta_{b} \ll r$, we can neglect the drift term (the second term) in the equation for $\vartheta$. Then we obtain:

$$
\vartheta-\iota \varphi=\text { const. }
$$

Let us expand the magnetic field in a Fourier series,

$$
B=\bar{B}\left[1+\frac{1}{2} \sum_{\mu, \nu} \epsilon_{B}^{(\mu \nu)} \exp (i \mu \vartheta-i \nu N \varphi)\right],
$$


where $\epsilon_{B}^{(\mu \nu)}=\epsilon_{B}^{(-\mu,-\nu)}$. Then, using equations (A.3) and (A.4) and neglecting terms of order $\left(\epsilon_{B}^{(\mu \nu)}\right)^{2}$, we can integrate equation (A.2). As a result, we obtain the following constant of the particle guiding centre (g.c.) motion:

$$
P^{g c}=\psi+\frac{B}{\omega_{B}} v_{\|} R_{0} \sum_{\mu, \nu} \frac{\mu \epsilon_{B}^{(\mu \nu)}}{\mu \iota-\nu N} \cos (\mu \vartheta-\nu N \varphi)=\mathrm{const},
$$

where $\nu>0$ or $\nu=0, \mu>0$.

It follows from equation (A.5) that when the dominant Fourier harmonics of the magnetic field are characterized by $\mu \sim 1$ and $N \gg 1$, the radial excursion of the particles is determined mainly by the harmonics with $\nu=0$ unless non-axisymmetric harmonics exceed the axisymmetric ones by a factor of $N$ or more. In particular, in Wendelstein-line stellarators the toroidal harmonic, $\epsilon_{B}^{(10)}$, determines the orbit width. Taking only the mentioned harmonic into account, we have:

$$
r=\bar{r}+\frac{r_{B \|}}{\iota} \frac{R_{0} \epsilon_{t}}{r} \cos \vartheta
$$

where $\epsilon_{t}=-\epsilon_{B}^{(10)}>0$ (in contrast to tokamaks, $\epsilon_{t} \neq r / R_{0}$ in stellarators). In the considered case of $N \gg 1$, harmonics with $\nu \neq 0$ lead only to relatively weak oscillations of the radial coordinate, although helical harmonics and/or the mirror harmonic typically exceed the toroidal harmonic (e.g., $\epsilon_{B}^{(-1,1)}>\epsilon_{t}$ in W7-AS, see figure 3).

Note that equation (A.6) can also be obtained from the conservation of the wellknown toroidal angular momentum, $P_{\varphi}^{g c}=\Psi_{p}-B_{\varphi} v_{\|} / \omega_{B}$, where the superscript " $\varphi$ " labels the corresponding covariant vector component. In addition, we note that, taking into account the finite Larmor radius, we have to replace $\Psi_{p}$ with $\Psi_{p}+\iota(\boldsymbol{v} \times \boldsymbol{B})^{\psi} /\left(B \omega_{B}\right)$. Then we obtain from $P_{\varphi}^{g c}$ the exact toroidal angular momentum, $P_{\varphi}=\Psi_{p}-v_{\varphi} B / \omega_{B}$.

These results can be generalized to particles located near the magnetic axis. Having omitted all harmonics except for $(\mu, \nu)=(1,0)$ in equation (A.4), one can easily check that $P^{g c}$ given by equation (A.5) [also with the harmonic $(\mu, \nu)=(1,0)$ only kept] is an invariant of equations (A.1). Introducing new "quasi-orthogonal" coordinates $x=r \cos \vartheta$ and $y=r \sin \vartheta$, we obtain

$$
P^{g c}=\frac{1}{2} \bar{B}\left[\left(x-\Delta_{b}\right)^{2}+y^{2}-\Delta_{b}^{2}\right]=\text { const. }
$$

Thus, the particle orbits on the $(x, y)$-plane are circles centered at $\left(\Delta_{b}, 0\right)$. Therefore, it is convenient to introduce the radial and angular coordinates associated with the circles, $\bar{r}$ and $\theta$, by the relationships $x=\Delta_{b}+\bar{r} \cos \theta$ and $y=\bar{r} \sin \theta$. Using equations (A.1) and (A.7), one can show that equations of motion take a very simple form in the new coordinates:

$$
\bar{r}=\text { const }, \quad \theta=\omega_{b}\left(t-t_{0}\right),
$$


where $t_{0}$ is an arbitrary constant, $\omega_{b}$ is the bounce frequency. Now we return to the coordinates $r$ and $\vartheta$. In the case when $\bar{r} \gg\left|\Delta_{b}\right|$, we recover equations (9) and (10). In the opposite case, when $\bar{r} \ll\left|\Delta_{b}\right|$ (i.e., for near-axis particles), we obtain

$$
\begin{aligned}
& r=\left|\Delta_{b}\right|+\bar{r} \cos \left[\omega_{b}\left(t-t_{0}\right)\right], \\
& \theta=\Theta+\frac{\bar{r}}{\left|\Delta_{b}\right|} \sin \left[\omega_{b}\left(t-t_{0}\right)\right],
\end{aligned}
$$

where $\Theta=0$ when $\Delta_{b}>0$ and $\Theta=\pi$ when $\Delta_{b}<0$.

\section{Appendix B. Features of the $\Phi_{m, n, l}$ harmonics}

The Fourier harmonics $\Phi_{m, n, l}$ associated with the finite orbit width are defined by

$$
\Phi_{m, n, l}(\bar{r})=\int_{-\pi}^{\pi} \frac{d \vartheta}{2 \pi} \Phi_{m, n}[r(\vartheta)] e^{-i l \vartheta},
$$

which can also be written in the form of equation (13). It is clear that $r=\bar{r}$ when $\Delta_{b}=0$. Therefore, it follows from equation (B.1) that $\Phi_{m, n, l=0}(\bar{r})=\Phi_{m, n}(r)$ and $\Phi_{m, n, l \neq 0}=0$ when the orbit width is negligible. In order to consider finite $\Delta_{b}$, we have to specify the mode radial structure. We take

$$
\Phi_{m, n}(r)=\hat{\Phi}_{m, n} \exp \left[-\left(r-r_{*}\right)^{2} / \Delta_{m}^{2}\right] .
$$

This Gaussian may approximate the mode structure of low-shear systems. This is shown in Appendix $\mathrm{C}$ for the gap modes. The GAE mode calculated for the Wendelstein 7AS shot \# 34723 can also be approximated by equation (B.2), see figure 7 . Using equation (B.2), we have $\Phi_{m, n}(r) \rightarrow \hat{\Phi}_{m, n} \sqrt{\pi} \Delta_{m} \delta\left(r-r_{*}\right)$ for $\Delta_{m} \rightarrow 0$. Then

$$
\Phi_{m, n, l}(\bar{r})=\hat{\Phi}_{m, n} \frac{\Delta_{m} \cos \left[l \cos ^{-1}\left(r_{*}-\bar{r}\right) / \Delta_{b}\right]}{\sqrt{\pi} \sqrt{\Delta_{b}^{2}-\left(r_{*}-\bar{r}\right)^{2}}} \propto \Delta_{m} \hat{\Phi}_{m, n}
$$

where $\max \left\{\left(r_{*}-\left|\Delta_{b}\right|\right),\left|\Delta_{b}\right|\right\} \leq \bar{r} \leq r_{*}+\left|\Delta_{b}\right|$. Equation (B.3) shows that $\Phi_{m, n, l=0}(\bar{r})$ is minimum at $\bar{r}=r_{*}$ and has two singularities at $\bar{r}=r_{*} \pm \Delta_{b}$ when $\left(r_{*}-\left|\Delta_{b}\right|\right)>\left|\Delta_{b}\right|$ [in contrast to the case of $\Delta_{b}=0$, when $\Phi_{m, n, l=0}(\bar{r})$ is a function with the only maximum at $\bar{r}=r_{*}$ ]. The singularities appeared because we used the approximation $\Phi_{m, n}(r) \propto \delta\left(r-r_{*}\right)$. The result of a calculation without this approximation is shown in figure 13(a). We observe that the increase of the ratio $\sigma \equiv\left|\Delta_{b}\right| / \Delta_{m}$ decreases the function "height" by a factor of $\sigma$ and spreads it by the same factor. This means that the integrals with the integrand proportional to $\Phi_{m, n, l=0}^{2}$ should decrease approximately as $\sigma^{-1}$ when $\sigma \gg 1$; the integrals decrease even stronger when $r_{*}<2\left|\Delta_{b}\right|$, figure 13(b). The matter is that the condition $\bar{r}>\left|\Delta_{b}\right|$ cuts the left-hand parts of the curves shown in figure 13(a) when $r_{*}$ approaches plasma centre. In fact, this figure is valid for $r_{*} \gg 2\left|\Delta_{b}\right|$ [or $r_{*}>2\left|\Delta_{b}\right|$ for $\left.\Phi_{m, n}(r) \propto \delta\left(r-r_{*}\right)\right]$. In particular, only the right-hand part of $\Phi_{m, n, l}(\bar{r})$, where $\bar{r}>r_{*}$, "survives" for $r_{*}=\left|\Delta_{b}\right|$.

The harmonics with $l \neq 0$ are considerable when $\sigma>1$. They are of the same order as the $l=0$ harmonic when $\sigma \gg 1$. However, the $l=0$ harmonic mainly contributes to 
the integral $\int \mathrm{d} \bar{r} \Phi_{m, n, l}^{2}$, see figure $13(\mathrm{~b})$, because, first, $\Phi_{m, n, l \neq 0}$ has zeroes and, second, the maximum of $\left|\Phi_{m, n, l \neq 0}(\bar{r})\right|$ is less than the maximum of $\left|\Phi_{m, n, l=0}(\bar{r})\right|$. Calculations of $\Phi_{m, n, l}$ for the GAE mode agree with these conclusions drawn for $\Phi_{m, n, l}$ described by equation (B.1), see figure 14.

Now we proceed to the particles that do not encircle the magnetic axis. For these particles, the dependence of $r$ on $\vartheta$ is not uniquely defined. Therefore, we will use the canonical angle,

$\theta=\omega_{\vartheta} t$, instead of the Boozer angle $\vartheta$. Then we obtain:

$$
\begin{aligned}
& \Phi_{m, n}[r(\theta)]=\sum_{l} \Phi_{m, n, l}^{0}(\bar{r}) e^{i l \theta}, \\
& \Phi_{m, n, l}^{0}(\bar{r})=\frac{1}{\pi} \int_{r_{\min }}^{r_{\max }} \mathrm{d} r \Phi_{m, n}(r) \frac{\cos \left(l \cos ^{-1} \frac{r-\left|\Delta_{b}\right|}{\bar{r}}\right)}{\sqrt{\bar{r}^{2}-\left(r-\left|\Delta_{b}\right|\right)^{2}}},
\end{aligned}
$$

where $r_{\min }=\left|\Delta_{b}\right|-\bar{r}$ and $r_{\max }=\left|\Delta_{b}\right|+\bar{r}$. One can see that the function $\Phi_{m, n, l}^{0}(\bar{r})$ has the following features. First, it is considerable provided that $r_{*}<2\left|\Delta_{b}\right|$ because $\bar{r} \leq\left|\Delta_{b}\right|$ for the semi-trapped particles. Second, it has a maximum at $\bar{r}=\left|r_{*}-\right| \Delta_{b}||\left[\Phi_{m, n l}^{0}(\bar{r})\right.$ is singular in this point when $\left.\Phi_{m, n}(r) \propto \delta\left(r-r_{*}\right)\right]$. Third, $\Phi_{m, n, l}^{0}(\bar{r}=|\Delta|)=\Phi_{m, n, l}(\bar{r}=|\Delta|)$ because equation (B.5) is transformed into equation (13) after the replacements $\bar{r} \rightarrow\left|\Delta_{b}\right|$ and $\left|\Delta_{b}\right| \rightarrow \bar{r}$. This implies that $\Phi_{m, n, l}^{0}(\bar{r})$ continuously extends the function $\Phi_{m, n, l}(\bar{r})$ to the region of $\bar{r}<\left|\Delta_{b}\right|$. In a particular case of $r_{*}=\Delta_{b}, \Phi_{m, n, l}^{0}(\bar{r})$ matches $\Phi_{m, n, l}(\bar{r})$ in the point where both these functions are minimum.

\section{Appendix C. The structure of gap Alfvén eigenmodes in low-shear configurations}

The aim of this Appendix is to study the radial structure and the eigenfrequencies of localized ideal Alfvén modes residing in gaps of the Alfvén continuum. The present analysis relies on the following assumptions. First, the magnetic shear, $\hat{s}$, satisfies the condition $\hat{s} \ll \epsilon \ll 1$, where $\epsilon$ is the characteristic magnitude of the coupling parameters that produce the mode (see below or the work [7] for the exact definition of the term "coupling parameters"). In this sense, our analysis is a generalization of that in the references $[26,27]$, where the multiplicity of TAE and EAE modes in lowshear configurations was discovered, to arbitrary continuum gaps. Second, the modes are sufficiently localized for the so-called "local approximation" (when the equilibrium quantities are considered to be constant) to be applicable. As we will see, in the limit case of $\hat{s} \ll \epsilon \ll 1$ the local approximation is applicable when $m \gg\left|\epsilon k_{\|} /(\hat{s} \iota)\right|^{1 / 2}$. Third, a mode consists mainly of two Fourier harmonics, the rest of the harmonics being negligible. Fourth, $\mathrm{d} \iota / \mathrm{d} r \neq 0$ in the mode localization region. Finally, we assume that the effect of the pressure gradient on the mode is negligible, and the aspect ratio of the device is large. 
Neglecting the effects of the plasma pressure and the plasma current (the latter does not affect strongly localized modes), we write the equation of ideal Alfvén eigenmodes as follows $[26,7]$ :

$$
\begin{aligned}
& \sum_{i, j}\left\{\frac{1}{g B^{2}} \hat{L} \frac{\partial}{\partial x^{i}}\left[\sqrt{g} g_{\perp}^{i j} B^{2} \frac{\partial}{\partial x^{j}}\left(\frac{\hat{L} \widetilde{\Phi}}{B^{2} \sqrt{g}}\right)\right]\right. \\
& \left.+\frac{1}{\sqrt{g}} \frac{\partial}{\partial x^{i}}\left(\sqrt{g} g_{\perp}^{i j} \frac{\omega^{2}}{v_{A}^{2}} \frac{\partial \widetilde{\Phi}}{\partial x^{i}}\right)\right\}=0,
\end{aligned}
$$

where $g_{\perp}^{i j}=g^{i j}-b^{i} b^{j} ; g^{i j}$ with $i, j=\psi, \vartheta, \varphi$ are the components of the contravariant metric tensor of Boozer coordinates $\left(x^{\psi}, x^{\vartheta}, x^{\varphi}\right)=(\psi, \vartheta, \varphi) ; b^{i}$ denotes the $i$-th contravariant component of $\boldsymbol{b}=\boldsymbol{B} / B ; g$ is the metric tensor determinant; $\hat{L}=$ $\iota \partial / \partial \vartheta+\partial / \partial \varphi$. In a large-aspect-ratio device, the components $g^{i \varphi}=g^{\varphi i}$ with $i=\psi, \vartheta, \varphi$ are small (of the order of the squared inverse aspect ratio) in comparison with the rest [7]; therefore, below we neglect the terms with $i=\varphi$ or $j=\varphi$ in equation (C.1).

To proceed, we normalize the metric coefficients:

$$
g^{i j}=\bar{g}^{i j}(\psi) h_{g}^{i j}(\psi, \vartheta, \varphi), \quad \frac{\sqrt{g}}{v_{A}^{2}} g^{i j}=c^{i j}(\psi) h_{c}^{i j}(\psi, \vartheta, \varphi),
$$

where $\bar{g}^{\psi \psi}=\left\langle g^{\psi \psi}\right\rangle,\langle(\ldots)\rangle=\oint \mathrm{d} \vartheta \mathrm{d} \varphi(\ldots) /\left(4 \pi^{2}\right), \bar{g}^{\psi \vartheta}=\bar{g}^{\psi \psi} /(2 \psi), \bar{g}^{\vartheta \vartheta}=\bar{g}^{\psi \psi} /\left(4 \psi^{2}\right)$, $c^{\psi \psi}=\left\langle g g^{\psi \psi}\right\rangle, c^{\psi \vartheta}=c^{\psi \psi} /(2 \psi), c^{\vartheta \vartheta}=c^{\psi \psi} /\left(4 \psi^{2}\right)$. Then we expand the angle-dependent factors in Fourier series as follows:

$$
h_{g, c}=\delta^{i j}+\frac{1}{2} \sum_{\mu, \nu=-\infty}^{\infty} \epsilon_{g, c(\mu, \nu)}^{i j} \exp (i \mu \vartheta-i \nu N \varphi)
$$

where $\delta^{i j}$ is the Kronecker symbol. The normalized Fourier coefficients $\epsilon_{g, c(\mu, \nu)}^{i j}$ will be referred to as "coupling parameters". We assume that $\epsilon_{g, c(-\mu,-\nu)}^{\psi \psi}=\epsilon_{g, c(\mu, \nu)}^{\psi \psi}$, $\epsilon_{g, c(-\mu,-\nu)}^{\vartheta \vartheta}=\epsilon_{g, c(\mu, \nu)}^{\vartheta \vartheta}, \epsilon_{g, c(-\mu,-\nu)}^{\psi \vartheta}=-\epsilon_{g, c(\mu, \nu)}^{\psi \vartheta}$, i.e., that $h_{g, c}^{\psi \psi}$ and $h_{g, c}^{\vartheta \vartheta}$ contain only cosine harmonics, and $h_{g, c}^{\psi \vartheta}$, only sine harmonics.

We further assume that the wave consists of only two Fourier harmonics with the poloidal and toroidal wave numbers $\left(m_{1}, n_{1}\right)$ and $\left(m_{2}, n_{2}\right)$, where $m_{2}=m_{1}+\mu_{0}$, $n_{2}=n_{1}+\nu_{0} N$, the integer parameters $\mu_{0}$ and $\nu_{0}$ (the "coupling numbers") determine the kind of the considered eigenmodes, for instance, $\left(\mu_{0}, \nu_{0}\right)=(1,0)$ for TAEs, $(2,0)$ for EAEs, $(0,1)$ for MAEs etc. Let $\psi=\psi_{*}$ be the radius where the longitudinal wave numbers of the two harmonics coincide, i.e.,

$$
\tilde{k}_{2}\left(\psi_{*}\right)=-\tilde{k}_{1}\left(\psi_{*}\right)=k_{*} \equiv\left(\mu_{0} \iota_{*}-\nu_{0} N\right) / 2 \text {, where } \tilde{k}_{i}=m_{i} \iota-n_{i}, \iota_{*}=\iota\left(\psi=\psi_{*}\right) .
$$
Following [26], we introduce new variables, $\Phi_{+}=\left(\Phi_{m_{1} n_{1}}+\Phi_{m_{2} n_{2}}\right) / 2$ and $\Phi_{-}=$ $\left(\Phi_{m_{1} n_{1}}-\Phi_{m_{2} n_{2}}\right) / 2$, instead of the harmonic amplitudes and a new radial variable, $z=-\left(\iota-\iota_{*}\right) \bar{m} /\left(\iota_{*} \hat{s}\right)$, where $\bar{m}=\left(m_{1}+m_{2}\right) / 2$, instead of $\psi$. Then we reduce equation (C.1) to a pair of equations for $\Phi_{+}$and $\Phi_{-}$. With this aim, we take the sum and the difference of the $\left(m_{1}, n_{1}\right)$ and $\left(m_{2}, n_{2}\right)$ harmonics of equation (C.1). Keeping only the main-order terms in $\bar{m}$ and omitting terms $\sim \hat{s}^{2}$, we obtain a pair of coupled 
equations, which can be symbolically united in the following single equation:

$$
\begin{aligned}
& \frac{\mathrm{d}}{\mathrm{d} z}\left\{\left[\Omega^{2}\left(1 \pm \frac{\epsilon_{c\left(\mu_{0}, \nu_{0}\right)}^{\psi \psi}}{2}\right)-k_{*}^{2}\left(1 \mp \frac{\epsilon_{g\left(\mu_{0}, \nu_{0}\right)}^{\psi \psi}}{2}\right)\right] \frac{\mathrm{d} \Phi_{ \pm}}{\mathrm{d} z}\right\} \\
& -\left[\Omega^{2}\left(1 \pm \frac{\epsilon_{c\left(\mu_{0}, \nu_{0}\right)}^{\vartheta \vartheta}}{2}\right)-k_{*}^{2}\left(1 \mp \frac{\epsilon_{g\left(\mu_{0}, \nu_{0}\right)}^{22}}{2}\right)\right] \Phi_{ \pm} \\
& \pm\left(i \epsilon_{c\left(\mu_{0}, \nu_{0}\right)}^{\psi \vartheta} \Omega^{2}+i \epsilon_{g\left(\mu_{0}, \nu_{0}\right)}^{\psi \vartheta} k_{*}^{2}\right) \frac{\mathrm{d} \Phi_{\mp}}{\mathrm{d} z} \\
& -2 k_{*} \hat{s} \iota\left[\frac{\mathrm{d}}{\mathrm{d} z}\left(z \frac{\mathrm{d} \Phi_{\mp}}{\mathrm{d} z}\right)-z \Phi_{\mp}\right]=0,
\end{aligned}
$$

where the first and second equations are obtained by taking the upper and lower signs, respectively, in the symbols " \pm " and "F", $\Omega=\omega R /\left\langle v_{A}\right\rangle, v_{A}$ is the Alfvén velocity, $R=\langle B\rangle\langle g\rangle^{1 / 2} \approx R_{0}$. For simplicity, we have neglected $\epsilon_{g(0,0)}^{\vartheta \vartheta}$ and $\epsilon_{c(0,0)}^{\vartheta \vartheta}$, which are small for large aspect ratio. In what follows, we will omit the subscripts " $\left(\mu_{0}, \nu_{0}\right)$ " when this does not lead to ambiguity.

The continuous spectrum of the obtained equations consists of the values of $\omega^{2}$ for which the matrix coefficient at second-order derivatives in equations (C.4) is degenerate (the solutions are singular). One can see that there is a gap in the continuum, which lies between $\Omega^{2}=k^{2}\left(1-\epsilon^{\psi \psi}\right)$ and $\Omega^{2}=k^{2}\left(1+\epsilon^{\psi \psi}\right)$, where $\epsilon^{i j}=\left(\epsilon_{g}^{i j}+\epsilon_{c}^{i j}\right) / 2$, and we have neglected terms $\sim \epsilon^{2}$, where, as mentioned above, $\epsilon$ is a small parameter showing the order of magnitude of the coupling parameters $\epsilon_{c, g\left(\mu_{0}, \nu_{0}\right)}^{i j}$. It will be convenient to introduce the normalized frequency, $g$, by the relationship $\Omega^{2}=k^{2}\left(1+\epsilon^{\psi \psi} g\right)$, so that the gap corresponds to $-1<g<1$.

Finally, to reduce the order of equations (C.4), we employ Fourier transformation. Expressing $\Omega^{2}$ in terms of $g$ and omitting terms $\sim \epsilon^{2}$, we obtain

$$
Z_{ \pm}(y) \Phi_{ \pm}=-2 i \Upsilon\left[\left(y^{2}+1\right) \frac{\mathrm{d} \Phi_{\mp}}{\mathrm{d} y}+y \Phi_{\mp}\right] \pm 2 \epsilon^{\psi \vartheta} y \Phi_{\mp},
$$

where $y$ is the wave number variable Fourier-conjugate to $z, \Upsilon=\iota \hat{s} / k_{*}$,

$$
Z_{ \pm}(y)=(g \pm 1) \epsilon^{\psi \psi} y^{2}+g \epsilon^{\psi \psi} \pm \epsilon^{\vartheta \vartheta} .
$$

Finding either $\Phi_{+}$or $\Phi_{-}$from one of equations (C.5) and putting it to the other one, we obtain a single second-order differential equation:

$$
\begin{aligned}
& 4 \Upsilon^{2} \frac{\mathrm{d}^{2} \Phi_{ \pm}}{\mathrm{d} y^{2}}=\frac{1}{\left(y^{2}+1\right)^{2}}\left[-Z_{+} Z_{-}\right. \\
& \left. \pm 4 i \epsilon^{\psi \vartheta} \Upsilon Z_{\mp} \frac{\mathrm{d}}{\mathrm{d} y}\left(\frac{y}{Z_{\mp}}\right)-4 y^{2}\left(\epsilon^{\psi \vartheta}\right)^{2}\right] \Phi_{ \pm},
\end{aligned}
$$

where we have omitted the terms $\propto \Upsilon^{2}$ except for the term containing $\mathrm{d}^{2} \Phi_{ \pm} / \mathrm{d} y^{2}$. However, a coefficient of the new equation contains $Z_{ \pm}(y)^{-1}$ and, thus, may become singular in the region of the mode localization when zeroes of $Z_{ \pm}(y)$ approach $y=0$. Therefore, one should express $\Phi_{+}$in terms of $\Phi_{-}$for $g<0$ and vice versa for $g>0$. Then (unless $\epsilon^{\psi \psi} \approx \epsilon^{\vartheta \vartheta}$ ) the denominators in the obtained equation can vanish only for $y \gg 1$. Thus, the signs in equation (C.7) correspond to $\operatorname{sgn}(g)= \pm 1$. 
Further analysis depends on relationships between coupling parameters. In a general case, following an analysis for TAE modes [26], we assume that the mode is localized so that $y \sim(\hat{s} / \epsilon)^{1 / 2}, \mathrm{~d} / \mathrm{d} y \sim(\hat{s} / \epsilon)^{-1 / 2}$. Then we keep only terms up to $\sim \epsilon \hat{s}$, transforming equation (C.7) to a Scrödinger equation:

$$
4 \Upsilon^{2} \frac{\mathrm{d}^{2} \Phi_{ \pm}}{\mathrm{d} y^{2}}=[-E+U(y)] \Phi_{ \pm}
$$

with the effective energy $E=g^{2}\left(\epsilon^{\psi \psi}\right)^{2}-\left(\epsilon^{\vartheta \vartheta}\right)^{2}$ and the effective potential

$$
U(y)=Q y^{2}
$$

where $Q=2\left[\epsilon^{\psi \psi} \epsilon^{\vartheta \vartheta}-\left(\epsilon^{\vartheta \vartheta}\right)^{2}-2\left(\epsilon^{\psi \vartheta}\right)^{2}\right]$. For localized solutions to exist, the coupling parameters must satisfy the inequality $Q>0$. If this is the case, the eigensolutions are similar to those for the TAE case [26]: there are two sequences of eigenvalues, $g_{+0}<g_{+1}<g_{+2}<\ldots<1$ and $g_{-0}>g_{-1}>g_{-2}>\ldots>-1$, at the edges of the gap. The eigenvalues $g_{ \pm l}$ are given by the following equation (cf. [26]):

$$
g_{ \pm l}= \pm\left|\frac{\epsilon^{\vartheta \vartheta}}{\epsilon^{\psi \psi}}\right|\left[1+\frac{2|\Upsilon| Q^{1 / 2}}{\left(\epsilon^{\vartheta \vartheta}\right)^{2}}(2|l|+1)\right]^{1 / 2}
$$

which implies that the eigenmodes exist only when $\left|\epsilon^{\vartheta \vartheta}\right|<\left|\epsilon^{\psi \psi}\right|$. However, equation (C.10) works only for low $|l|$ : when $|l|$ is too high, the eigenvalues are outside the gap; in addition, the eigenfunctions for high $|l|$ are less localized than it was assumed in our derivation. The eigenfunctions are the well-known wave functions of the quantum oscillator. Having in mind that $\Phi_{\mp} \sim(\hat{s} / \epsilon) \Phi_{ \pm} \ll \Phi_{ \pm}$[see equation (C.5)], we find that the eigenfunctions corresponding to $g_{ \pm l}$ look in real space as follows:

$$
\Phi_{m_{1} n_{1}} \approx \mp \Phi_{m_{2} n_{2}} \propto \exp \left[-\frac{m^{2}\left(r-r_{*}\right)^{2}}{2 A^{2} r_{*}^{2}}\right] H_{|l|}\left[-\frac{m\left(r-r_{*}\right)}{A r_{*}}\right],
$$

where $H_{l}(x)$ is the Hermite polynomial (in particular, $H_{0}(x)=1$ ), $A=2^{-1 / 2}|\Upsilon|^{-1 / 2} Q^{1 / 4}$. We observe that the characteristic width of the mode is $\Delta_{m} \sim\left[\left|\epsilon k_{*} /(\hat{s} \iota)\right|^{1 / 2} / m\right] r_{*}$, whence the condition of the applicability of the local approximation is $m \gg\left|\epsilon k_{*} /(\hat{s} \iota)\right|^{1 / 2}$.

The potential (C.9) is degenerate in the special case of $Q \approx 0 . \quad Q \approx 0$ is a relationship between $\epsilon_{\left(\mu_{0}, \nu_{0}\right)}^{\psi \psi}, \epsilon_{\left(\mu_{0}, \nu_{0}\right)}^{\psi \vartheta}$, and $\epsilon_{\left(\mu_{0}, \nu_{0}\right)}^{\vartheta \vartheta}$, which holds, in particular, at the magnetic axis for $\left(\mu_{0}, \nu_{0}\right)=(0,0),(2,0)$, and $(2,1)$ (i.e., for core-localized MAE, EAE, and $\mathrm{HAE}_{21}$ modes), as follows from analytical expressions for the metric tensor [7]. In this case, one needs to involve higher-order terms in equation (C.7), and the analysis becomes rather complicated. An exception is the case when $\epsilon^{\vartheta \vartheta}=-\epsilon^{\psi \psi}(1-c)$ with $c \sim Q \sim \hat{s} / \epsilon$. The above analysis indicates that then we should assume that $g= \pm(1-d)$, $d \sim \hat{s} / \epsilon, y \sim(\hat{s} / \epsilon)^{1 / 4}$, and $\mathrm{d} / \mathrm{d} y \sim(\hat{s} / \epsilon)^{-1 / 4}$. Keeping terms up to $\sim \epsilon^{2}(\hat{s} / \epsilon)^{3 / 2}$ in equation (C.7), we arrive at equation (C.8) but with $E=2(c-d)\left(\epsilon^{\psi \psi}\right)^{2} \mp 4 i \Upsilon \epsilon^{\psi \vartheta}$, $U(y)=\left(Q \mp 4 i \Upsilon \epsilon^{\psi \vartheta}\right) y^{2}$. We infer that there may be two sequences of the eigenvalues, $g_{+0}<g_{+1}<g_{+2}<\ldots<1$ and $g_{-0}>g_{-1}>g_{-2}>\ldots>-1$,

$$
g_{ \pm l}= \pm\left[1-c+\frac{|\Upsilon|}{\left(\epsilon^{\psi \psi}\right)^{2}}\left(Q \mp 4 i \Upsilon \epsilon^{\psi \vartheta}\right)^{1 / 2}(2|l|+1)\right]+\frac{2 i \Upsilon \epsilon^{\psi \vartheta}}{\left(\epsilon^{\psi \psi}\right)^{2}}
$$


However, either sequence really exists only when $\left|g_{ \pm 0}\right|<1$ and $Q \mp 4 i \Upsilon \epsilon^{\psi \vartheta}>0$. For instance, for EAE and $\mathrm{HAE}_{21}$ modes, the coupling parameters at the magnetic axis satisfy the relationships $\epsilon^{\psi \psi}=-\epsilon^{\vartheta \vartheta}=-i \epsilon^{\psi \vartheta}$, whence $Q=0, c=0$. As follows from equation (C.12), only one of $g_{ \pm 0}$ is within the gap. It is located at the upper edge of the gap $\left(g_{ \pm 0} \epsilon^{\psi \psi}>0\right)$ when $\hat{s} / k_{*}>0$ and vice versa. Thus, we recover the conclusion that EAEs $\left(k_{*}>0\right)$ in tokamaks with positive low shear appear at the upper edge of the EAE frequency gap [27]. However, when shear is negative, which is more typical in stellarators, EAEs occupy the lower tip of the gap. The HAE $\mathrm{H}_{21}$ modes $\left(k_{*}=\iota-N / 2<0\right)$ have the opposite properties: they reside at the upper edge of the gap when $\hat{s}<0$ and at the lower edge when $\hat{s}>0$. The eigenmodes corresponding to $g_{ \pm l}$ are also given by equation (C.11) (up to corrections $\sim(\hat{s} / \epsilon)^{1 / 4}$ ) but with $A=2^{-1 / 2}|\Upsilon|^{-1 / 2}\left(Q \mp 4 i \Upsilon \epsilon^{\psi \vartheta}\right)^{1 / 4}$.

Another important case is $\epsilon^{\psi \psi}=\epsilon^{\vartheta \vartheta}, \epsilon^{\psi \vartheta}=0$, which corresponds to core-localized MAEs. In this case equations (C.5) possess no localized solutions. As shown numerically and analytically [10], ideal MAEs owe their existence to the pressure gradient, which is disregarded in our analysis.

\section{References}

[1] Fu G.Y., Cheng C.Z. 1992 Phys. Fluids B 43722

[2] Breizman B.N., Sharapov S.E. 1995 Plasma Phys. Control. Fusion 371057

[3] Fülöp T. et al 1996 Plasma Phys. Control. Fusion 38811

[4] Gorelenkov N.N., Cheng C.Z., Fu G.Y. 1999 Phys. Plasmas 62802

[5] Wagner F. 1998 Transactions of Fusion Technology 3367

[6] Nakajima N., Cheng C.Z., Okamoto M. 1992 Phys. Fluids B 41115

[7] Kolesnichenko Ya.I., Lutsenko V.V., Wobig H., Yakovenko Yu.V., Fesenyuk O.P. 2001 Phys. Plasmas 8491

[8] Nührenberg C. 1999 Plasma Phys. Control. Fusion 411055

[9] Weller A. et al 2001 Phys. Plasmas 8931

[10] Lutsenko V.V., Kolesnichenko Ya.I., Weller A., Werner A., Wobig H., Yakovenko Yu.V., and Fesenyuk O.P. 2002 in Fusion Energy 2002 (Proc. 19th Int. Conf. Lyon, 2002) (Vienna: IAEA) CD-ROM file and http://www.iaea.org/programmes/ripc/physics/fec2002/html/fec2002.htm

[11] Toi K., Takechi M., Isobe M. et al 2000 Nucl. Fusion 401349

[12] Toi K., Yamamoto S., Nakajima N. et al 2004 Plasma Phys. Control. Fusion 46 S1

[13] Kolesnichenko Ya.I., Lutsenko V.V., Wobig H., Yakovenko Yu.V. 2002 Phys. Plasmas 9517

[14] Kolesnichenko Ya.I., Yakovenko Yu.V., Weller A., Werner A., Geiger J., Lutsenko V.V., Zegenhagen S. 2005 Phys. Rev. Lett. 94165004

[15] Yakovenko Yu.V., Kolesnichenko Ya.I., Lutsenko V.V., Weller A., Werner A., Zegenhagen S., Geiger J. 2004 in Fusion Energy 2004 (Proc. 20th Int. Conf. Vilamoura, 2004) (Vienna: IAEA) CD-ROM file and http://www-naweb.iaea.org/napc/physics/fec/fec2004/datasets/index.html

[16] Kolesnichenko Ya.I., Yamamoto S., Yamazaki K., Lutsenko V.V., Nakajima N., Narushima Y., Toi K., Yakovenko Yu.V. 2004 Phys. Plasmas 11158

[17] Ehmler H., Baldzuhn J., McCormick K., Kreter A., Klinger T., W7-AS Team 2003 Plasma Phys. Control. Fusion 4553

[18] Fesenyuk O.P., Kolesnichenko Ya.I., Lutsenko V.V., Wobig H., Yakovenko Yu.V. 2003 Proc. 30th EPS Conf. on Controlled Fusion and Plasma Physics (St. Petersburg, Russia, 2003) vol 27A (ECA) CD-ROM file, P-3.4

[19] Kolesnichenko Ya.I., Lutsenko V.V., White R., Yakovenko Yu.V. 2000 Phys. Rev. Lett. 842152

[20] Boozer A.H. 1981 Phys. Fluids 241999 
[21] Hirshman S.P., Lee D.K. 1986 Comput. Phys. Comm. 39161

[22] Nührenberg J., Zille R. Equilibrium and Stability of low shear stellarators Proc. Joint VarennaLausanne Workshop on Theory of Fusion Plasmas (Varenna)(Editrice Compositori Bologna, 1988) p 3

[23] Schwab C. 1993 Phys. Fluids B 53195

[24] Hirshman S.P., van Rij W.I., Merkel P. 1986 Comput. Phys. Commun. 43143

[25] Kolesnichenko Ya.I., Lutsenko V.V., Werner A., Wobig H., Geiger J., Burdo O.S. 2004 Proc. 31 st EPS Conf. on Plasma Physics (London, UK, 2004) vol 28G (ECA) CD-ROM file, P-1.201

[26] Candy J., Breizman B.N., Van Dam J.W., Ozeki T. 1996 Phys. Letters A 215299

[27] Sharapov S.E., Mikhailovskii A.B. 1997 Proc. 5th IAEA Tech. Comm. Mtg on Alpha Particles in Fusion Research (Abingdon, September 1997) (Abingdon: Joint European Torus) p 129 


\section{Figure captions}

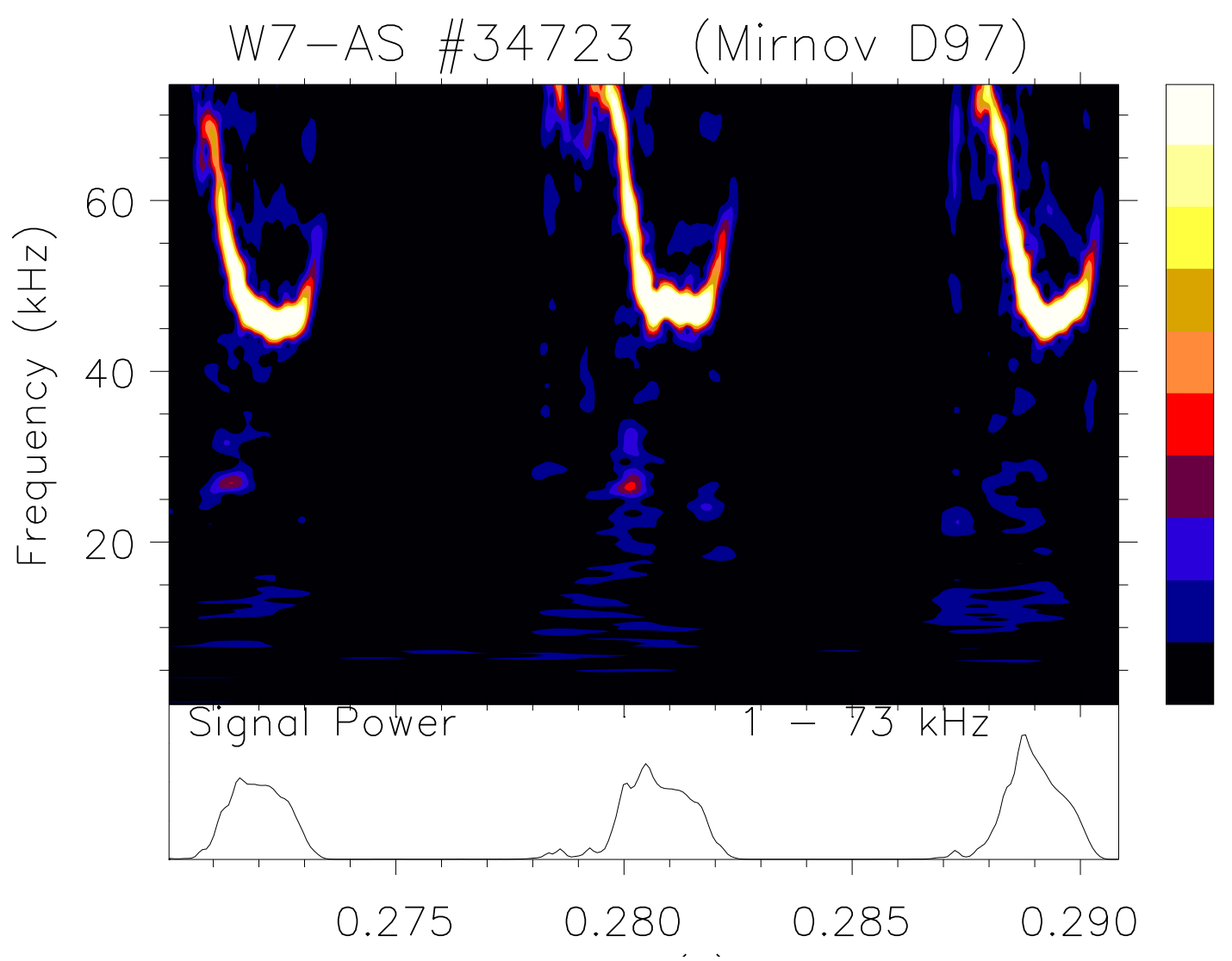

Figure 1. Bursts of Alfvén instability in the W7-AS shot \#34723. 


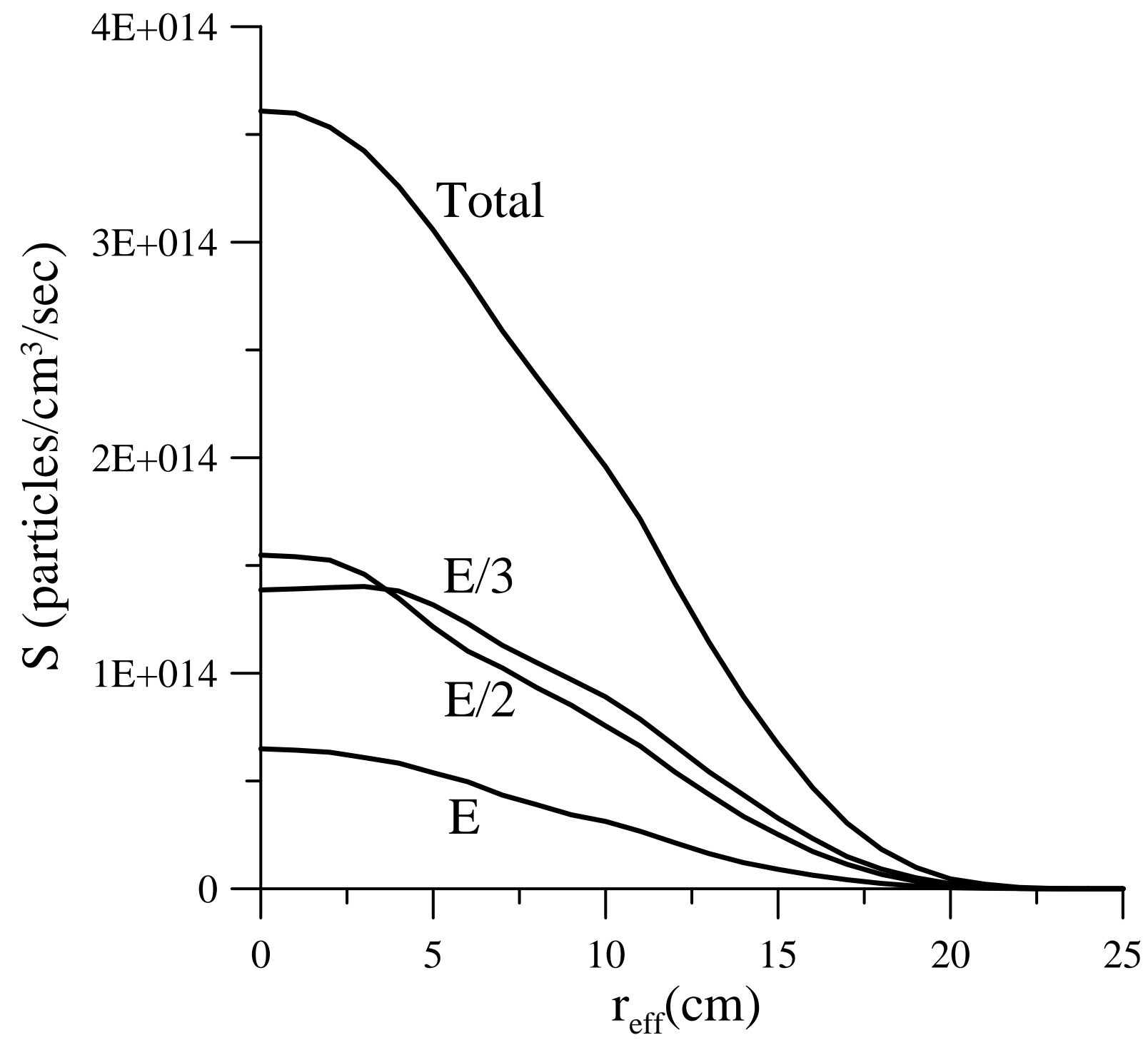

Figure 2. Birth profiles of the injected ions. 

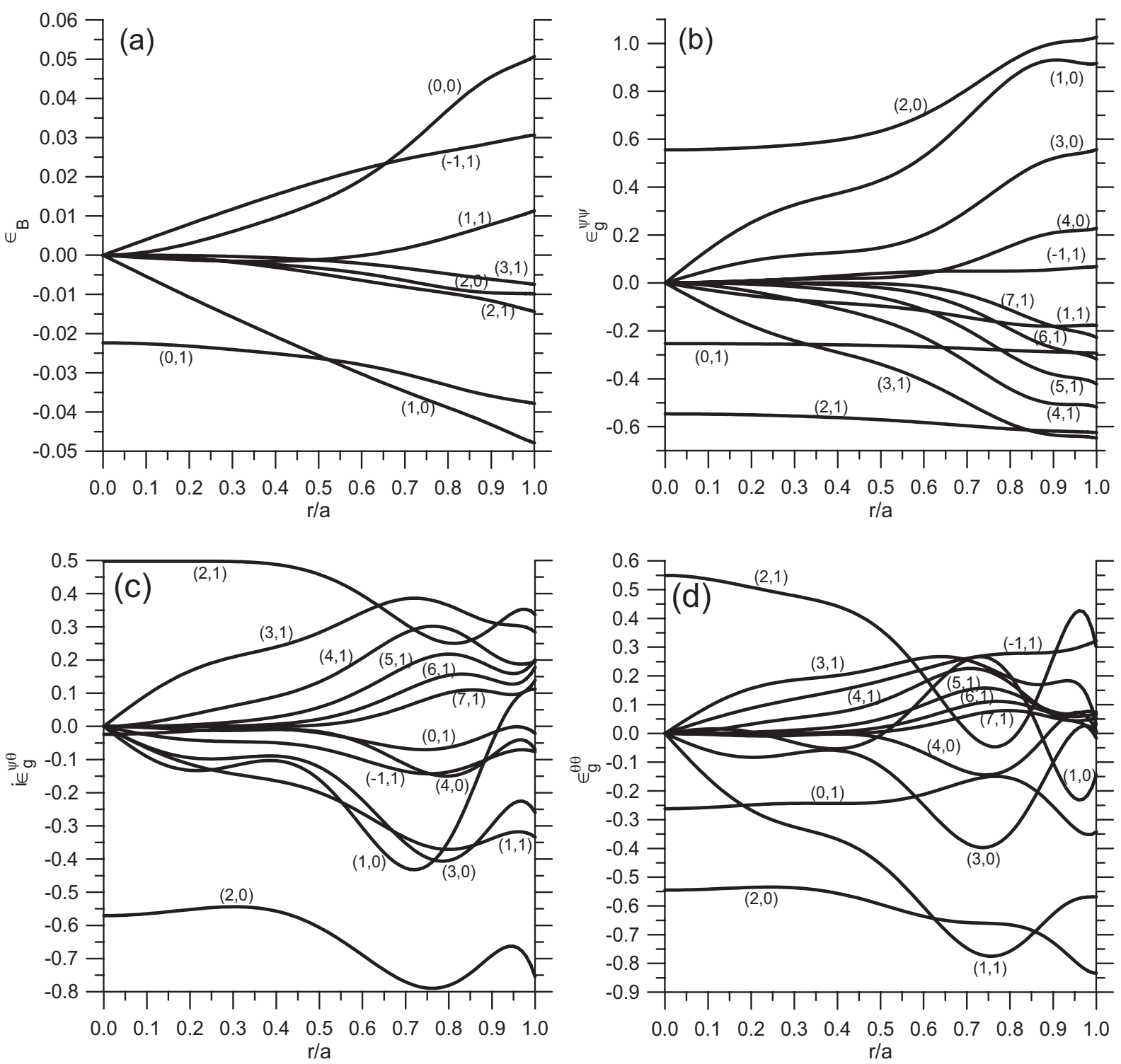

Figure 3. Fourier harmonics of the magnetic field strength $\left(\epsilon_{B}\right)$ and the metric tensor $\left(\epsilon_{g}\right)$ in the W7-AS shot \#34723 at $t=0.29$ s (i.e., immediately after a burst of Alfvénic activity). 


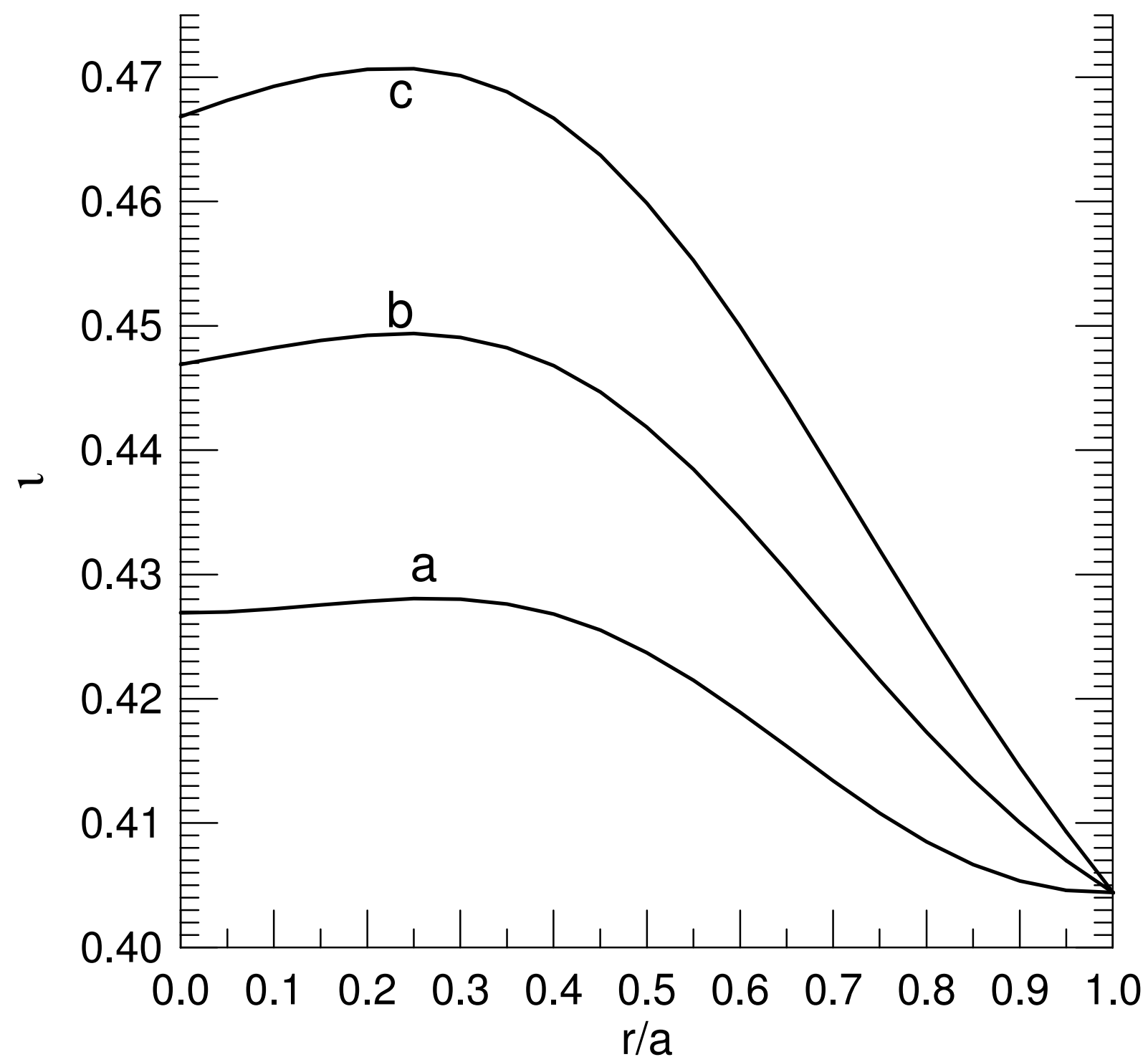

Figure 4. The rotational transform in the W7-AS shot \#34723 at $t=0.29$ : a, calculated by NEMEC [24]; b, adopted for description of the final stage of the instability bursts; c, adopted for description of the initial stage of the instability bursts. The corrections are made by taking into account the calculated Alfvén continuum branches. 

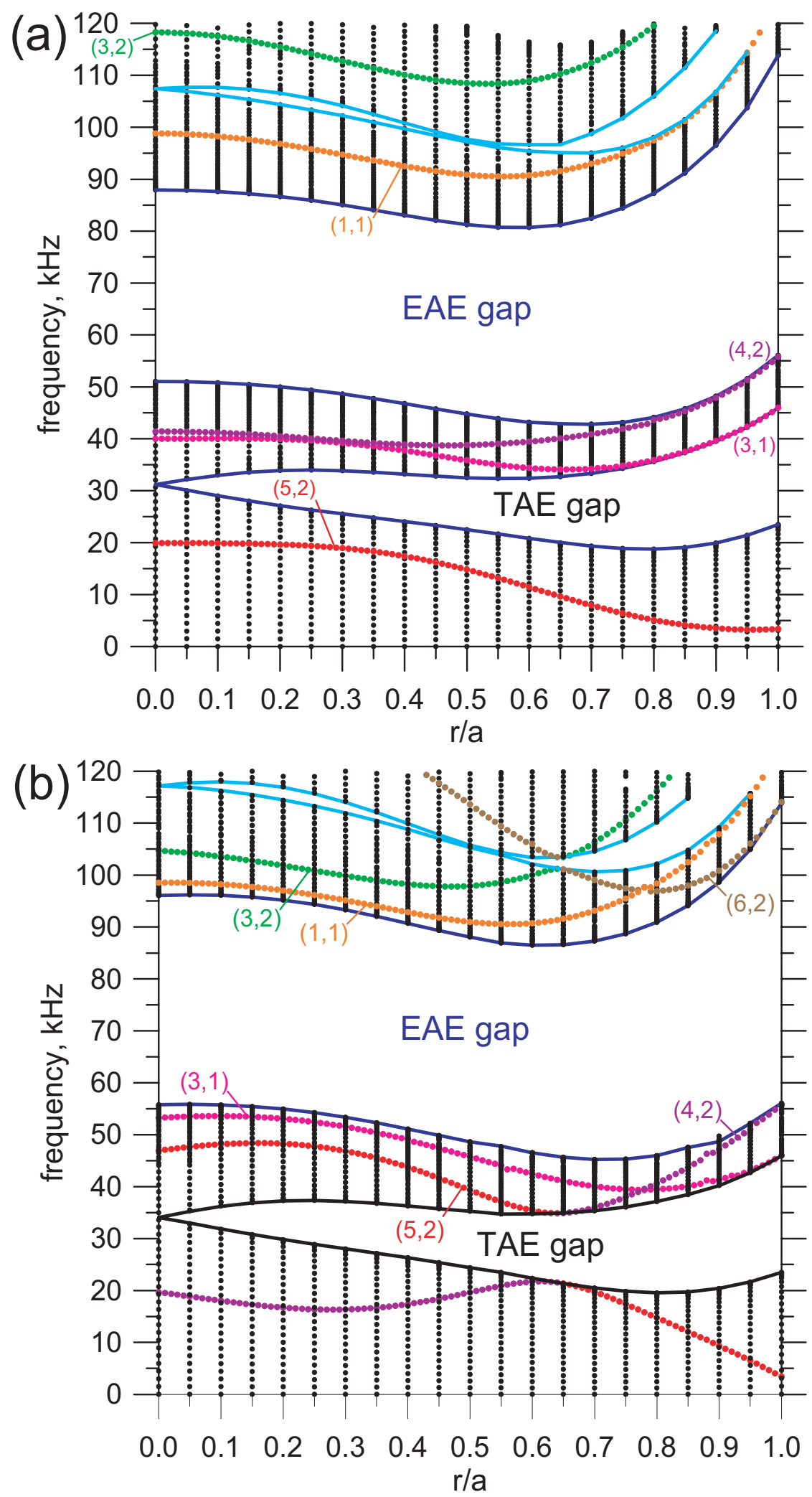

Figure 5. Alfvén continuum for the W7-AS shot \#34723 at $t=0.29$ : a, the case of $\iota_{*}=0.427 ; \mathrm{b}$, the case of $\iota_{*}=0.471$. 


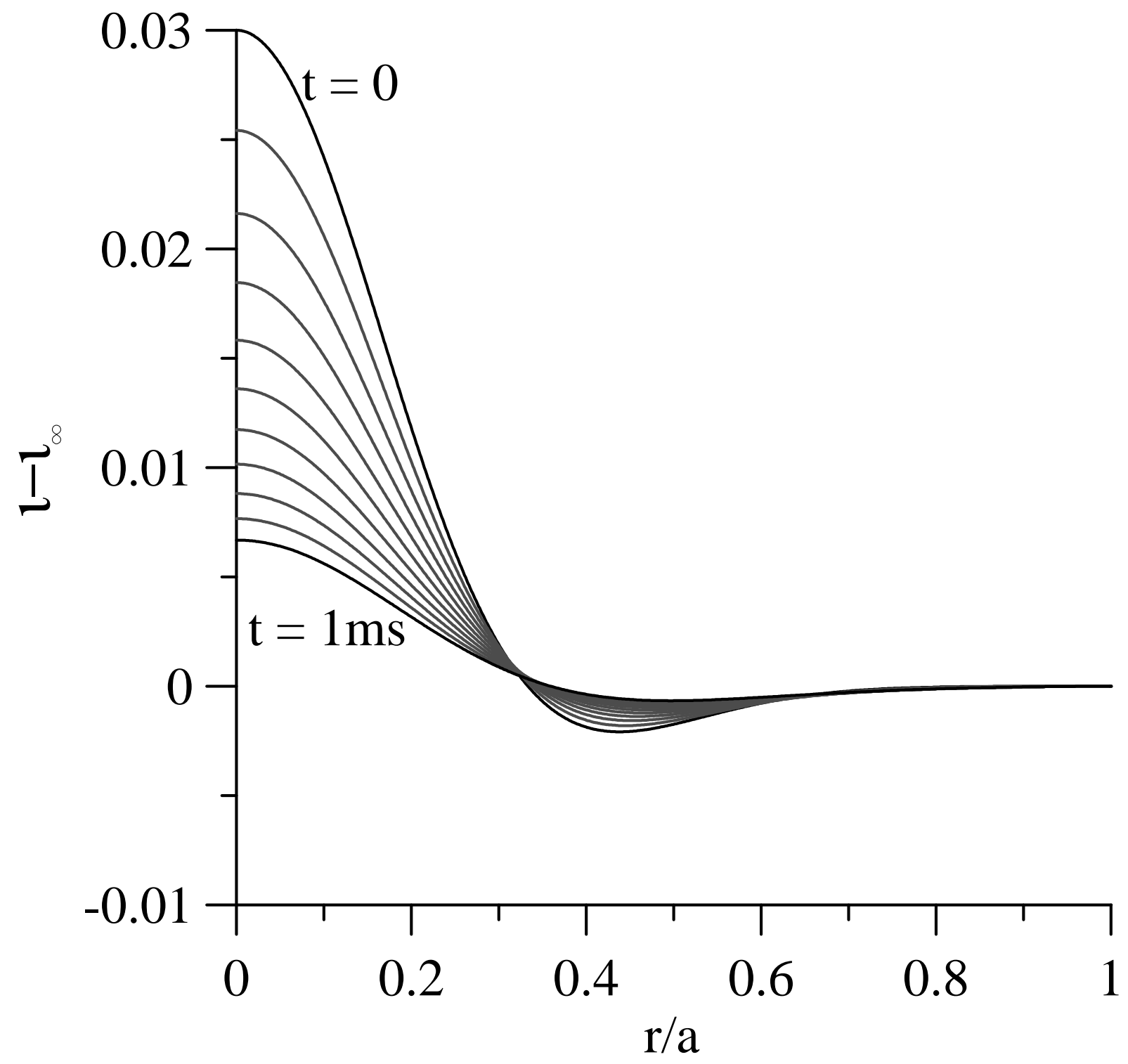

Figure 6. Possible evolution of the rotational transform during a burst of the Alfvén instability. 


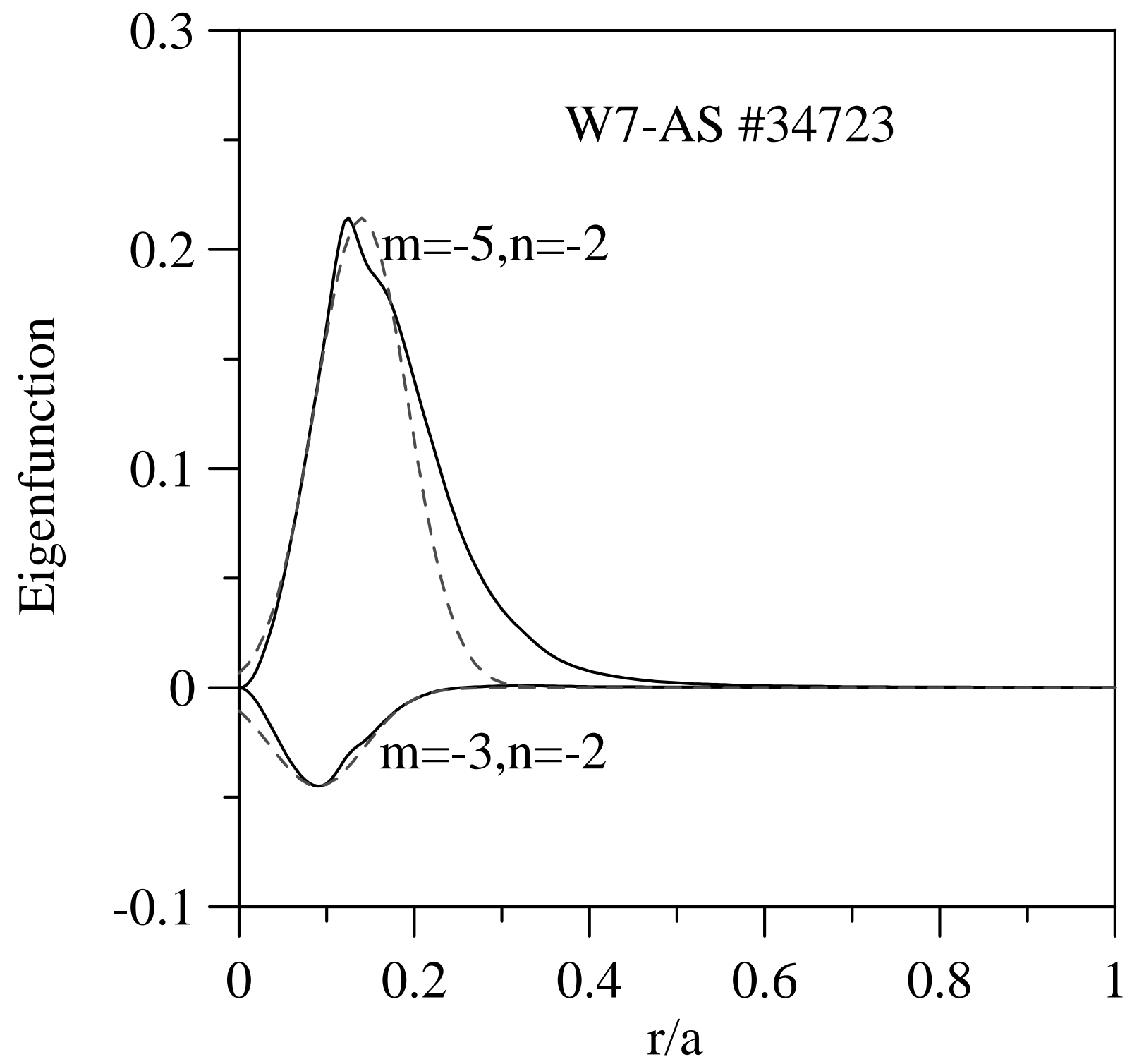

Figure 7. Scalar potential of an Alfvén eigenmode calculated with the code BOA$\mathrm{E}$ [10] for the final stage of the instability bursts in the W7-AS shot \#34723. Dotted line shows the mode structure approximated as $\left.\Phi_{m, n} \propto \exp \left[-\left(r-r_{*}\right)\right]^{2} / \Delta_{m}^{2}\right]$ with $r_{*}=2.1 \mathrm{~cm}$ for $m=-5, r_{*}=1.35 \mathrm{~cm}$ for $m=-3$ and $\Delta_{m}=1.1 \mathrm{~cm}$. 


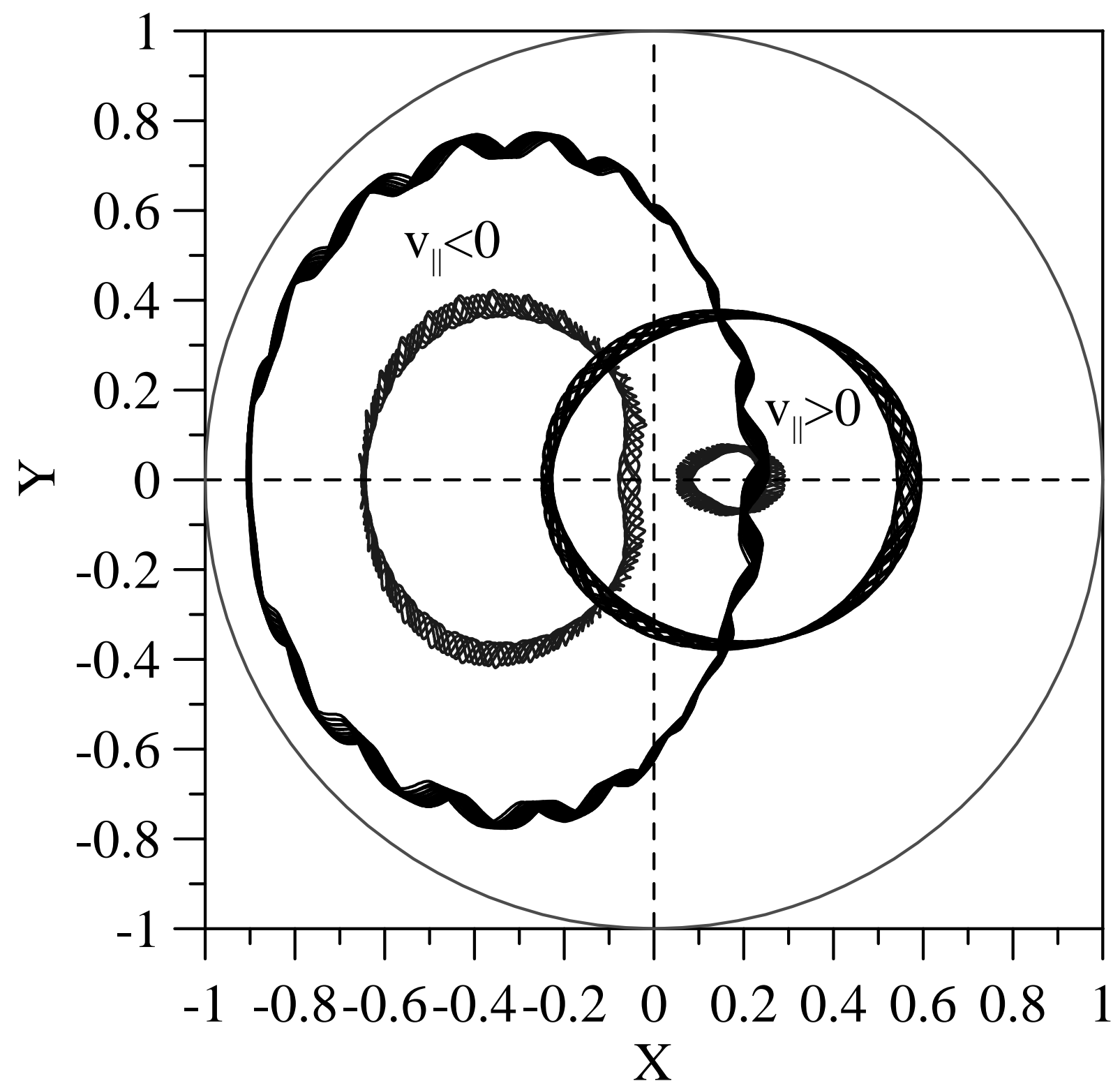

Figure 8. Orbits of injected ions with $\mathcal{E}=48 \mathrm{keV}$ and $|\chi| \equiv\left|v_{\|}\right| / v=1$ in the W7AS shot \#34723. Notations: $X=(r / a) \cos \vartheta, Y=(r / a) \sin \vartheta$, where $r$ is defined by $\psi=\bar{B} r^{2} / 2, \vartheta$ is the Boozer poloidal angle. Both standard (encircling the magnetic axis) and semi-trapped orbits (not encircling the axis) are shown. The orbits are calculated with the code ORBIS [25]. 


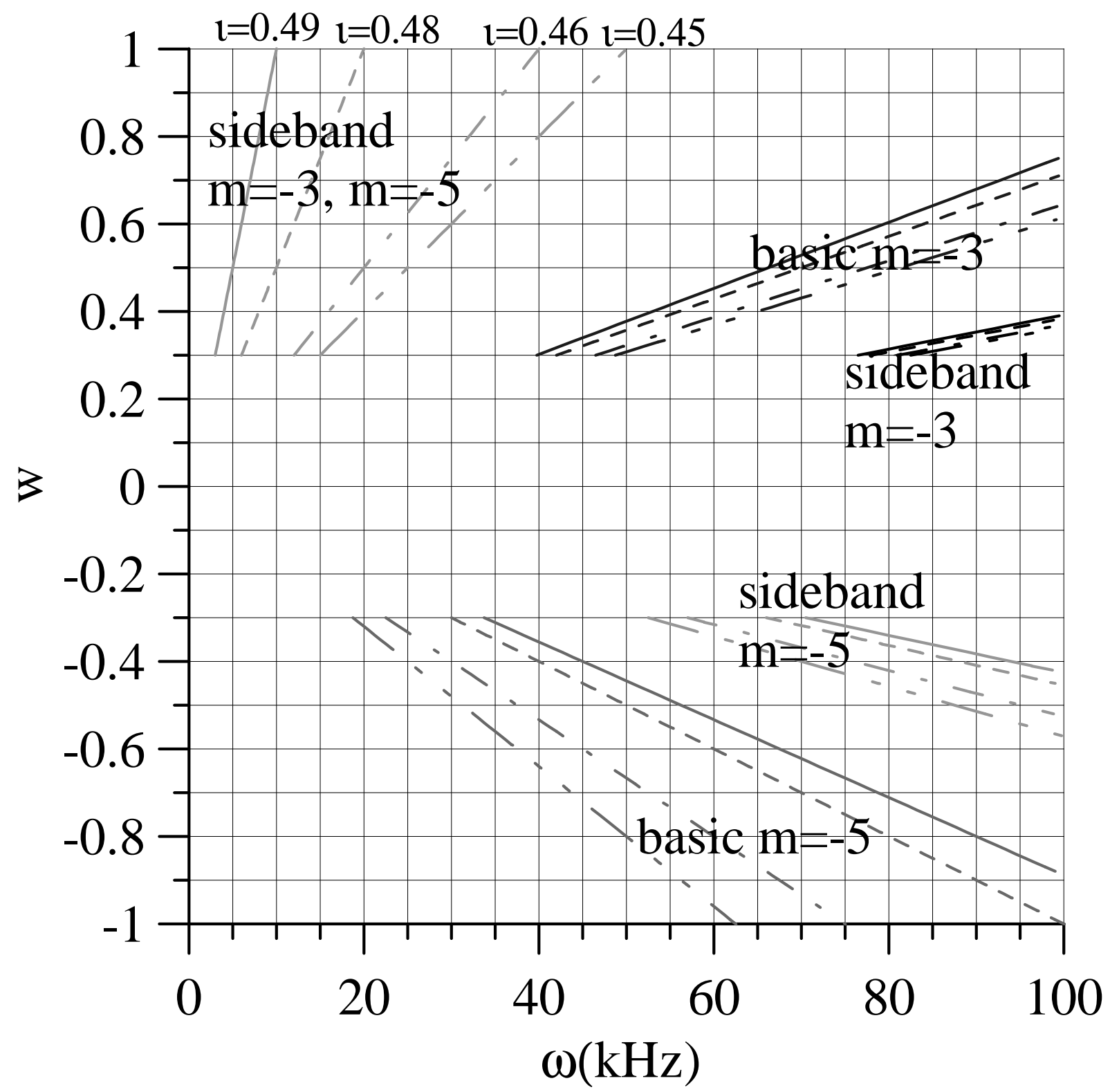

Figure 9. The resonant velocities, $w \equiv v_{\|} / v_{0}$, determined by the sideband resonance (39) and the basic resonance $\omega=k_{\|} v_{\|}$versus $\omega$ for various $\iota_{*}$ when the dependence of $\omega$ on $\iota$ is neglected. 


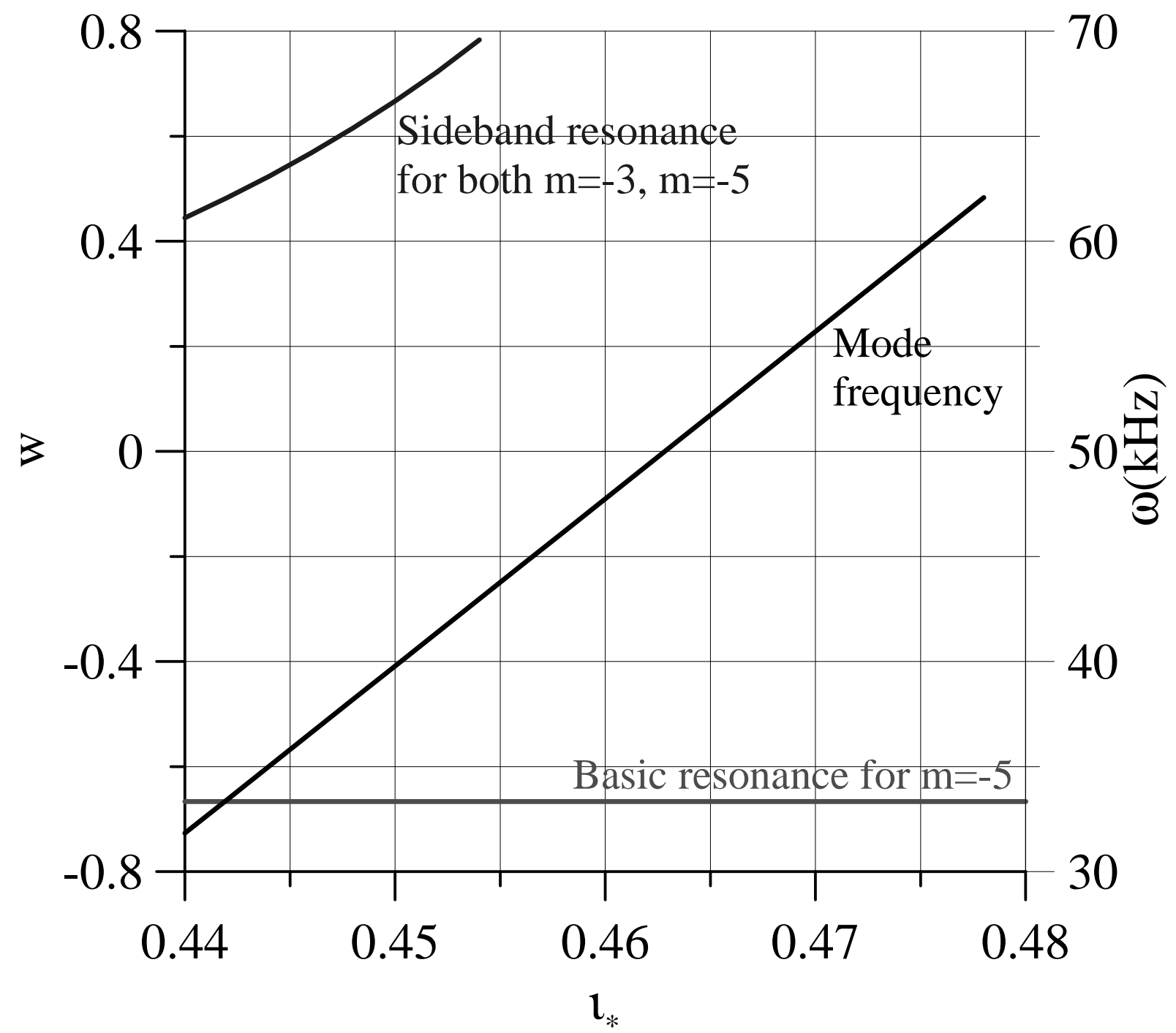

Figure 10. The frequency and the resonant velocities determined by the sideband resonance (39) and the basic resonance $\omega=k_{\|} v_{\|}$versus $\iota_{*}$ when $\omega=k_{\|}\left(\iota_{*}\right) v_{A}$. 

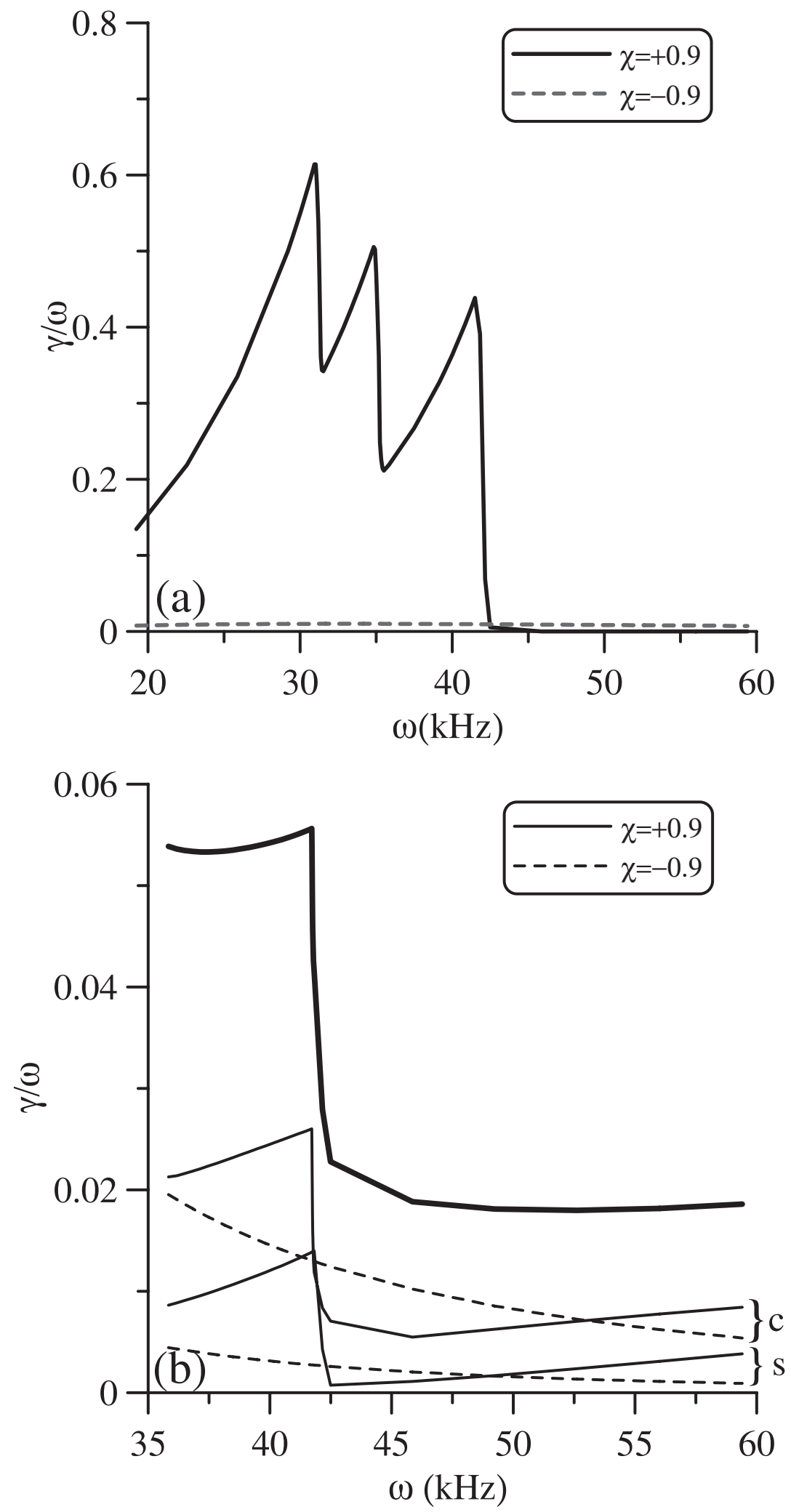

Figure 11. The growth rate of the instability in the W7-AS shot \#34723: a, the orbit width is neglected; b, the orbit width is taken into account. Symbol "c" labels circulating particles, symbol "s" labels semi-trapped particles. 

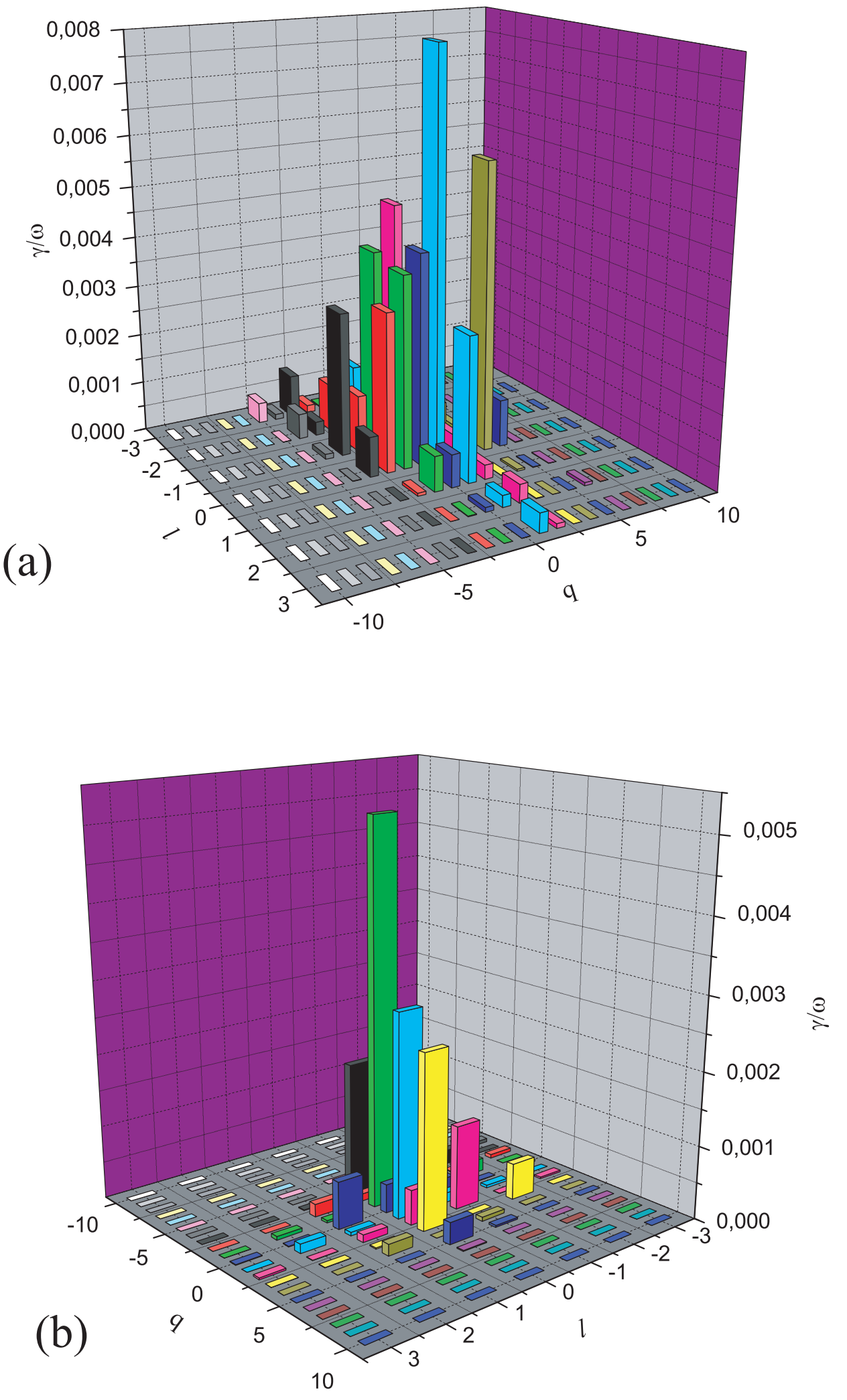

Figure 12. The growth rates associated with various individual resonances: a), $\omega=40 \mathrm{kHz} ; \mathrm{b}), \omega=60 \mathrm{kHz}$. 

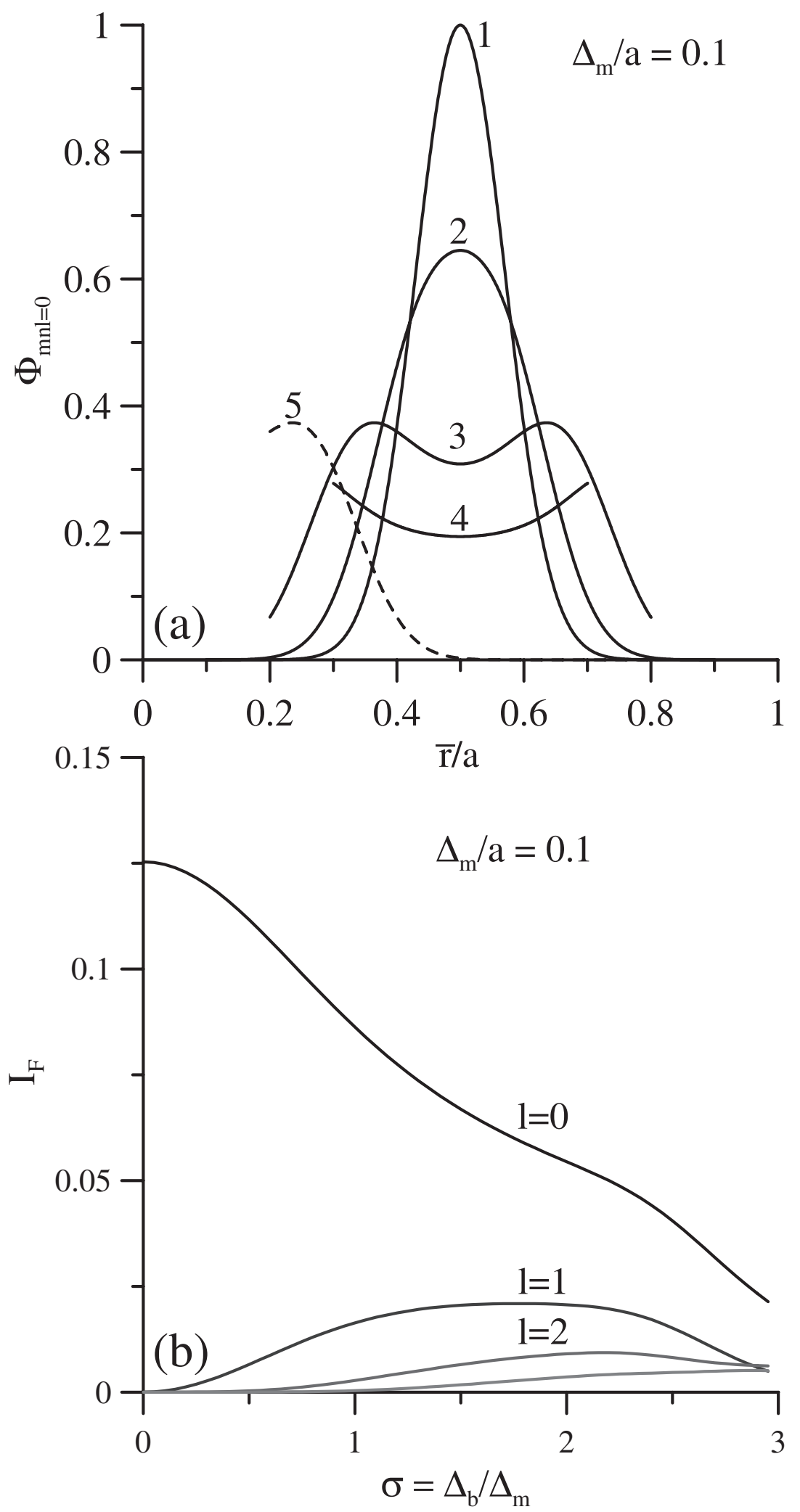

Figure 13. Features of Fourier harmonic defined by equation (B.1) for $\Phi_{m, n}=$ $\hat{\Phi}_{m, n} \exp \left(-x^{2}\right)$ with $x=\left(r-r_{*}\right)^{2} / \Delta_{m}^{2}$ : a, the radial structure of the $l=0$ harmonic for various $\sigma \equiv \Delta_{b} / \Delta_{m} ; 1, \sigma=0.001 ; 2, \sigma=1 ; 3, \sigma=2 ; 4, \sigma=3 ; 5, \sigma=2, r_{*}=0.1$; $\mathrm{b}$, the integral $I_{F}=\int d \bar{\rho}\left|\Phi_{m, n, l}\right|^{2}$ versus $\sigma$ for various $l$. 


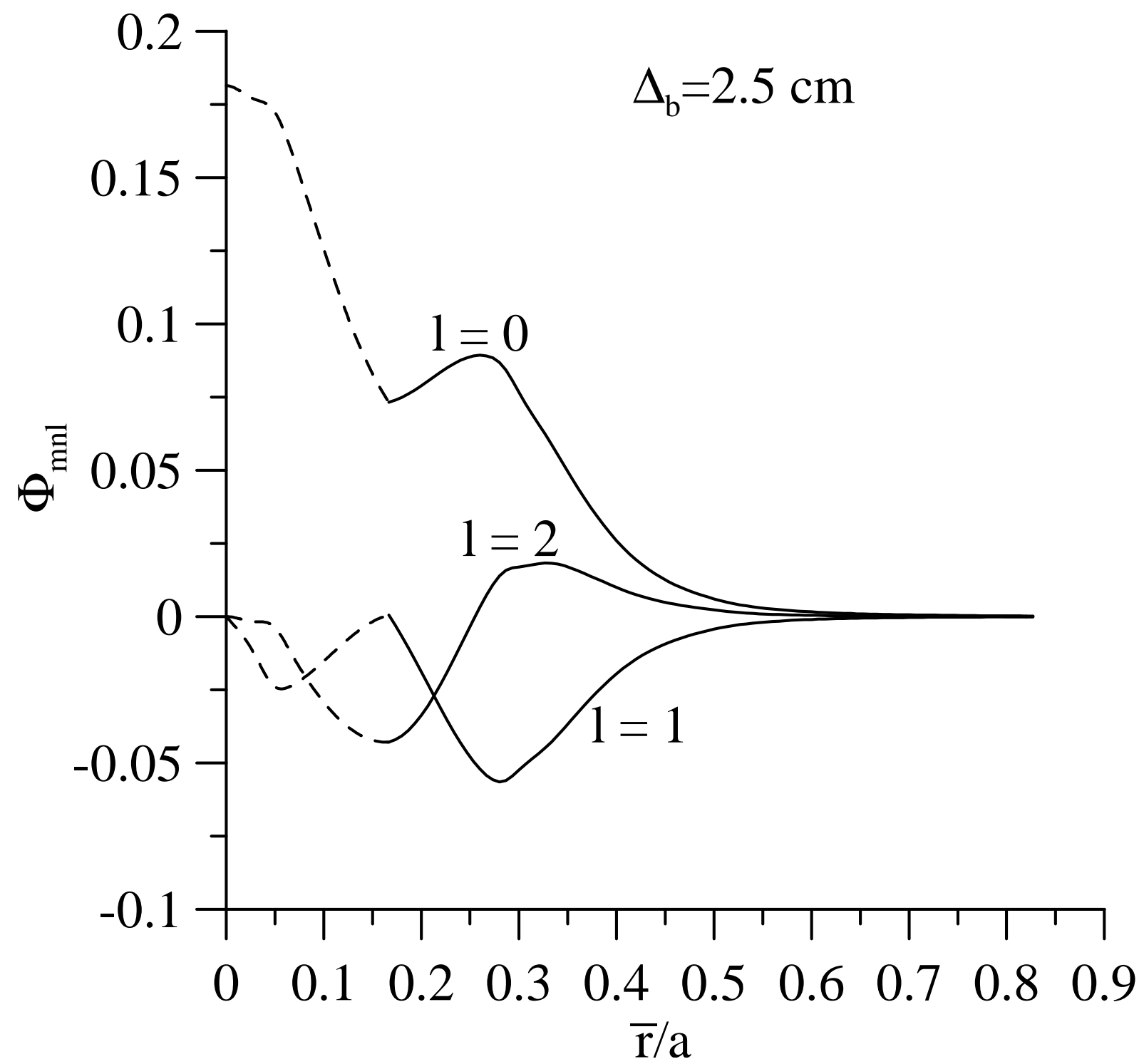

Figure 14. The radial structure of the $\Phi_{m, n, l}$ harmonics for various $l$ in the case of the eigenmode shown in figure 7 . Solid lines correspond to circulating particles; dotted lines, to semi-trapped particles. 